\title{
Electromechanical Characterization and Locomotion Control of IPMC BioMicroRobot
}

\author{
Martin J.-D. Otis \\ Applied Sciences Department, REPARTI Center, University of Quebec at Chicoutimi, Chicoutimi, QC, Canada G7H $2 B 1$ \\ Correspondence should be addressed to Martin J.-D. Otis; martin_otis@uqac.ca
}

Received 22 June 2013; Accepted 6 September 2013

Academic Editor: Kean Aw

Copyright (C) 2013 Martin J.-D. Otis. This is an open access article distributed under the Creative Commons Attribution License, which permits unrestricted use, distribution, and reproduction in any medium, provided the original work is properly cited.

\begin{abstract}
This paper presents the electromechanical characterization of Nafion-Pt microlegs for the development of an insect-like hexapod BioMicroRobot (BMR). BMR microlegs are built using quasi-cylindrical Nafion-Pt ionomeric polymer-metal composite (IPMC), which has 2.5 degrees of freedom. The specific manufacturing process using a laser excimer for one leg in three-dimensional configurations is discussed. Dynamic behavior and microleg characteristics have been measured in deionized water using a laser vibrometer. The use of the laser vibrometer shows the linear characteristics between the duty cycle of square wave input and displacement rate of the actuator at multiple frequencies. This linearity is used to design a servo-system in order to reproduce insect tripod walking. As well, BMR current consumption is an important parameter evaluated for each leg. Current passing throughout the IPMC membrane can result in water electrolysis. Four methods are explained for avoiding electrolysis. The hardware test bench for measurements is presented. The purpose of this design is to control a BMR for biomedical goals such as implantation into a human body. Experimental results for the proposed propulsion system are conclusive for this type of bioinspired BMR.
\end{abstract}

\section{Introduction}

Microrobotic development in submillimeter size has really appeared with MEMS (Microelectromechanicals systems), using the MUMP process (Multiuser MEMS processes) and microfabrication process with excimer laser and LIGA (Lithographie, Galvanoformung, and Abformung) microfabrication technology [1]. Several designers and researchers in micro- and nanorobotics hope their robots will perform biomedical applications. Currently, these robots are in the early stages of development, and the objectives to be reached are spread out over several decades [2]. Micro- and nanorobotics are an important area covering the knowledge of science and applied science in robotics and microsystems. Natural law explains that the smaller a living organism is, the harder it is to kill it. BioMicroRobot is designed with this simple rule. If the control of micro- and nanotechnology is possible, it will be also possible to easily help or to kill living organisms (cancer, bacteria, etc.), as well as organic or inorganic substances (kidney stones).

Many microactuators are in development for insect-like microrobot legs, such as bimorph cantilever structures acting as thermal actuators coupled or not with photonic band gap (PBG) materials, piezoelectric, electrostatic, magnetostrictive, shape memory alloy (SMA) and electroactive polymer (EAP) such as ionic polymers to name a few [3]. Some approaches are very interesting, but in biomedical applications, greater problems involve material biocompatibility. This constraint limits the use of some inert materials, largely used in human body like platinum, NiTi (Nitinol), some stainless steels, ceramic alumina, and some polymers. Materials from a biological source are interesting for microrobotic applications. This material should work overall in robot mission time without degradation, corrosion, or wear in the chemical environment (enzyme, $\mathrm{pH}$, and under lymphocyte action). Of course, this material must not be toxic to the host system [4].

BioMicroRobot (BMR) is a microrobot used to help living organisms such as the human body in its metabolism and to resolve health problems like kidney stones. It can be used to understand, explore, change, or control biological systems. BMR with biosensors can replace medical instrumentation for monitoring and measuring physiological events such as an in vivo glucose biosensor with hydrogel. 
BMR propulsion needs specific studies considering its many constraints. In this order of size and magnitude, it is necessary to study the context of its use and the BMR environment (aqueous medium, its conductivity and velocity, ambient temperature, pressure, etc.). Manufacturing is also an important challenge because of three dimensional handling of independent microparts. With the power supply being limited, it is necessary to reduce current consumption while optimizing mechanical properties of the actuator (force and velocity).

This paper covers all IPMC actuator parameters to be optimized for use on a BMR. Based on the choice made for the design of the microleg, it will be possible to manufacture it. The first part of the paper presents the chemical and mechanical manufacturing process of the microleg, which is critical for good quality and electromechanical properties. A failure in the procedure and bad handling could damage actuators and decrease performance. Indeed, metal electrode distribution particles added at the membrane surface are very sensitive to the chemical conditions of treatment, impregnation, and reduction. Second, for reducing some disadvantaged electromechanicals properties, a detailed model is carried out. Lastly, this paper recommends a central pattern generator for locomotion control using the actuator model. Displacement rate according to pulse width modulation (PWM) duty cycle and the resonance frequency coupled with a fluid according to the length is presented. This relationship will be very important for the design of a bioinspired servosystem in order to control the BMR like an insect in two- or three-dimensional environments.

1.1. Previous Work. Microrobot projects coming from Shuxiang Guo's laboratory are particularly advanced in designing microrobot with IPMC actuator. Recently, they study the position accuracy using 8 legged-locomotion using an IPMC model for one motion direction (using two electrodes on each surface) [5]. Also, other studies relates IPMC position control $[6,7]$ and in-pipe microrobot [8]. However, these microrobots are designed in order to move along a plane and to float in suspension without maneuverability in three-dimension.

This paper proposes a BMR similar to an insect with six microlegs. In order to move the body in a three-dimensional environment, each leg has to move in at least three axes (three degrees of freedom or $3 \mathrm{DOF}$ ). Since the robot is limited in size, power consumption, and manufacturing complexity, this paper suggests to design a leg which has one axis of movement coupled with the others two. Therefore, this configuration is considered as a minimum, which correspond to 2.5 degrees of freedom motion. This configuration allows driving the BMR as an insect tripod. However, with a lower DOF, the use of the push-slip method is also a design option such as piezoceramic actuation on a plane surface [9], but this propulsion reduces the application to a rigid surface. Higher DOF is better when the propulsion surface is deformed and many obstacles are present. To avoid obstacles, microlegs must leave the surface, which explained the need for 2.5 DOF per microleg.

Moreover, the actuator must have sufficient dimensions to support a microrobot of millimeter-length size. Along these lines, previous paper was devoted to the manufacturing process of tubular and fiber IPMC $[10,11]$. A clear explanation on the manufacturing process of the four electrode microlegs using a laser is provided to allow actuation with several degrees of freedom. Starting with some samples, it will be possible to measure the electromechanical characteristics quantitatively.

1.2. BioMicroRobot Geometry and Design. The BMR microactuator consists of six (6) quasi-cylindrical Nafion-Pt ionomeric polymer-metal composites (IPMC), and one tubular IPMC connected to a micropump enabling thrust vector control. IPMC has three (3) components: an ion-exchange membrane (IEM) like the perfluorosulfonic acid polymer, some cations, and at least two electrodes. Using a 6-legged locomotion is generally used to improve the stability of the microrobot during a displacement on a flat surface such as the cockroach tripod walking. However, some applications where more complex maneuvers in fluid such as obstacle avoidance and trajectory following require navigation in three dimensions enabling swimming capabilities. In this situation, it would be possible to use a thrust vector controlled by a tubular IPMC. First, a micropump installed on the back of the robot pushes the ambient fluid in a tubular IPMC. The curved tube directs the fluid jet generating a thrust (force) vector located at the IPMC anchored point which push microrobot following Newton's laws of motion. The use of a micropump is however necessary with the tube, which still represents a significant technological challenge. The characterization of this system (micropump, IPMC, and tubular fluid density) is not a part of this paper.

IEM consists of a cross-linked ionomer matrix with relatively uniform distribution of ion-active sites (cluster network) throughout the structure. The effects of different types of cations, electrode-membrane interfacial areas, and surface electrode resistance on performance are well known [12-14].

Each IPMC component has an effect on the electromechanical characteristics. IPMC works on two principles. First, the electroosmosis in the ionomer moves the cation and water to the cathode side by the electrostatic force when an electric field is applied. The osmotic pressure inside the polymer creates a bending that produces a corresponding displacement [15]. The second principle is the pumping effect of the cations. It would be the dominant contribution in the case of hydrophobic cations. It seems that the superposition effect works with different cation types, such as the ammonium form and alkali cations [16].

Our BMR design is presented in Figure 1. A vision system is also currently being evaluated, which is designed like an echosounder. Since the leg design should move in 2.5 degrees of freedom, the microleg tip movement could be represented by Figure 2. The motion of the microleg is composed of two gait phases: swing phase (recovery phase) and stance phase (support the weight body). The anterior transition point (transition from swing to stance) is called the anterior extreme position (AEP), and the posterior transition point is called the posterior extreme position (PEP).

This electroactive polymer or IPMC actuator has many advantages over all others; it has a quick response to low 


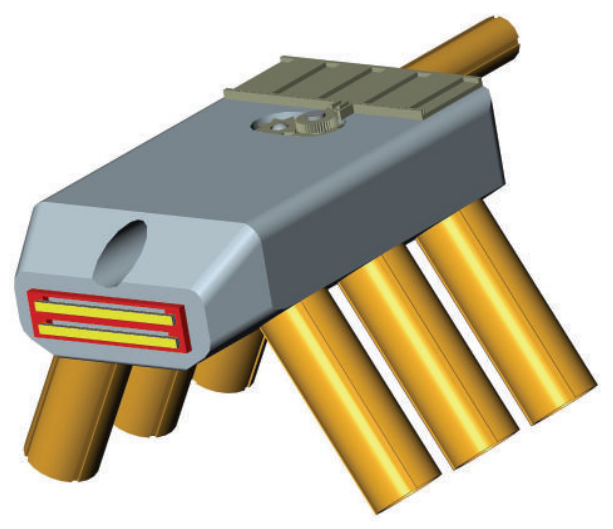

(a)

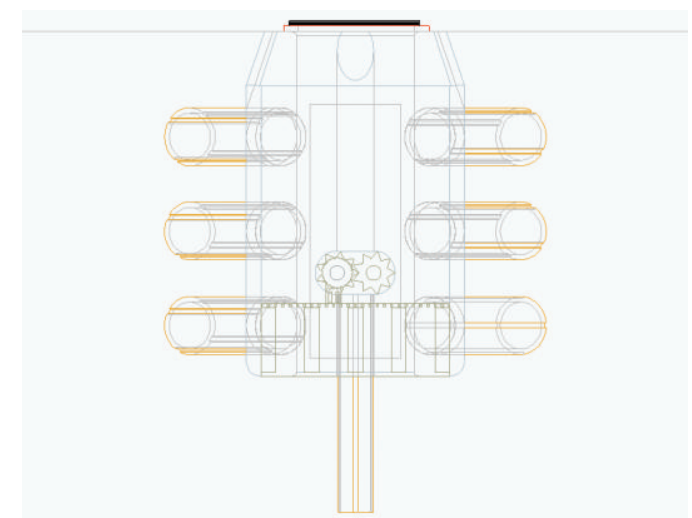

(b)

FIGURE 1: BioMicroRobot geometry using IPMC micropump and six IPMC microlegs.

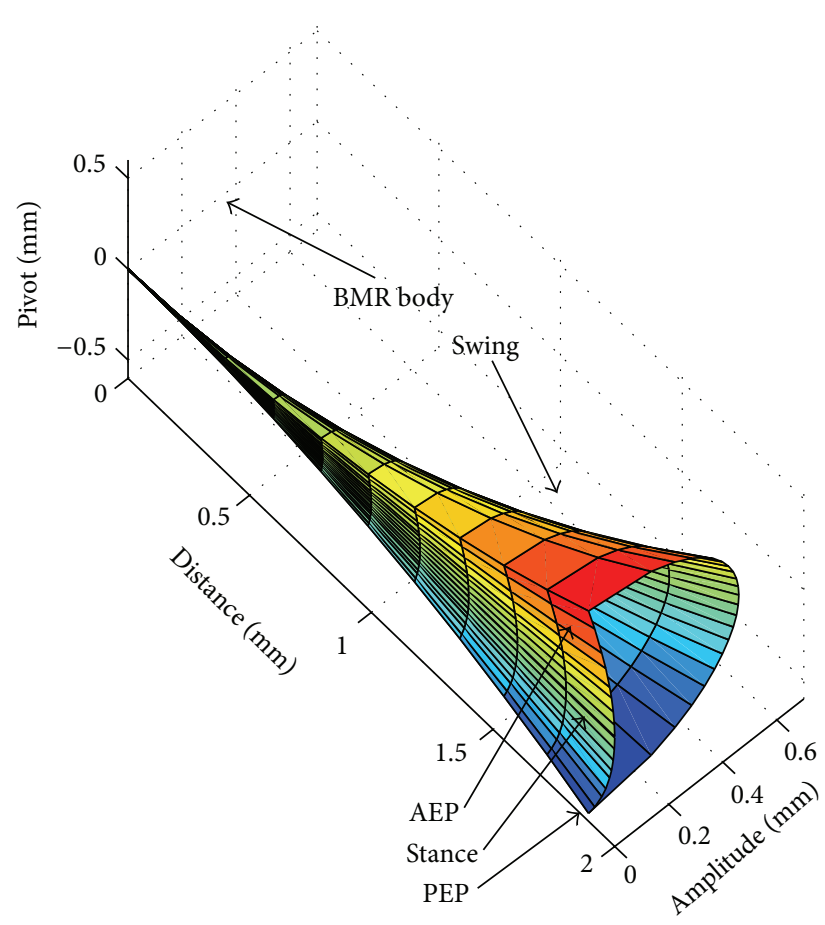

FIGURE 2: Ideal microleg tip movement in 2.5 DOF.

voltage, bends more than $100 \mathrm{~K}$ times, generates distributive deformation, has a low weight, can be downsized easily, and can be used in water [17]. It seems to be safe in the human body [18] if no water electrolysis is produced. It can be driven at low voltage, less than 2 volts. However, as described in Figure 3, we have measured a step transient response showing high instantaneous current consumption with Nafion-Pt and Lithium counter ion.

Water electrolysis and current consumption are two major IPMC disadvantages for microrobot applications. In part of this paper, we will demonstrate how to avoid electrolysis of Nafion-Pt IPMC with $\mathrm{Li}^{+}$. Platinum dissolution is also an inevitable consequence of metal use in the stimulation regime. Dissolved platinum is toxic in living organisms [19].

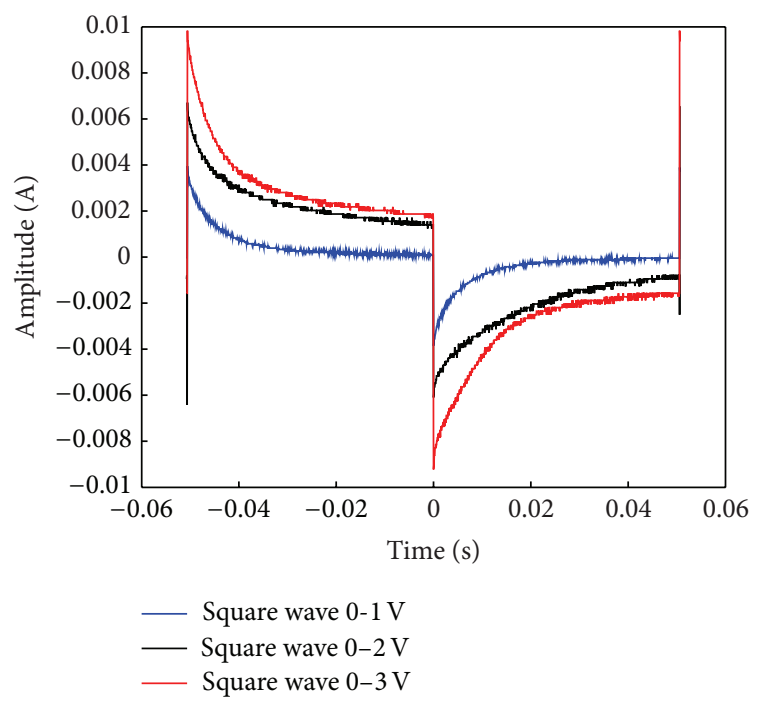

FIGURE 3: Current response to different squares waves voltage input.

\section{Materials and Methods}

In previous studies, we have used the tubular Perma Pure Inc. TT-030 Nafion [10] for the micropump design. Actuator movement is adjusted to orient the fluid direction. For a microleg design, the tubular shape generates too small displacement for this application. In another application, Nafion tube will be used to create an implantable inductive coil for BMR telemetry and power supply. In fact, the use of Nafion N-117 (DuPont) fiber (quasi-cylindrical cross section) is better for the displacement rate and current consumption [11]. The size of this new microactuator is about $200 \mu \mathrm{m}$ $\times 200 \mu \mathrm{m}$. Its length $L$ is about $4,000 \mu \mathrm{m}$ for the actuator characterization. Expected final microleg length on the BMR body is about $1,500 \mu \mathrm{m}$.

2.1. IPMC Manufacturing. This method can be done using any ionomer membrane. Ion exchange membrane (IEM) is a hydrophilic ionic polymer, which swells up about $10 \%$ in water at room temperature. The membrane $\mathrm{N}-117$ is cut into 


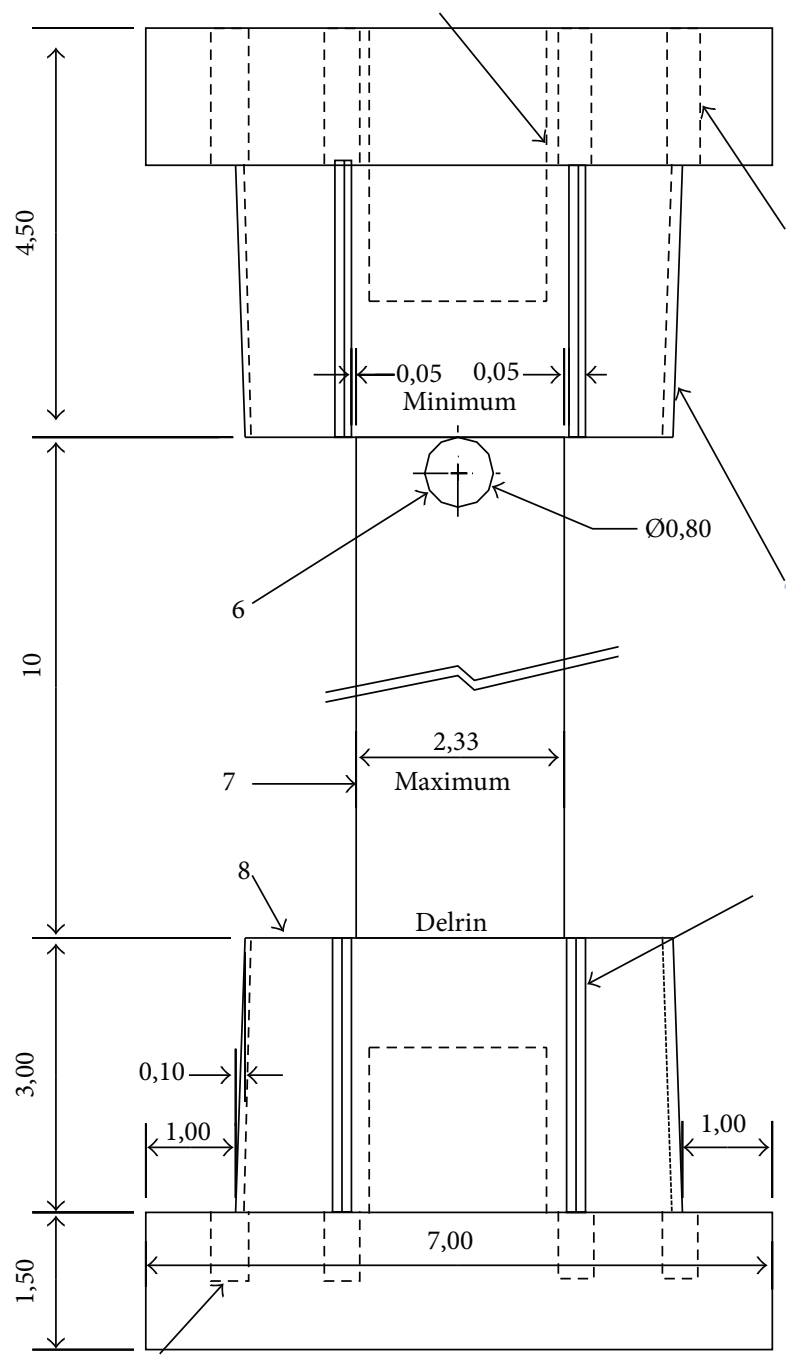

FIGURE 4: Six fiber holders for the manufacturing process.

fibers of $187 \mu \mathrm{m}$ with a sliding microtome LEICA SM 2500. The speed of microtome blade is set to $0.5 \mathrm{~mm} /$ second with an angle of attack set at about -2 degrees. Next, the six fibers are attached to a homemade support as shown in Figure 4.

The ionomer is cleaned in an ultrasonic water bath and boiled in $\mathrm{HCl}$ and water for about 30 minutes each. The ionomer is chemically plated with platinum using the impregnation and reduction (IR) process [20] as shown in Figure 5. Ion exchange (absorption in Figure 5(a)) and ion reduction (Figure 5(b)) are repeated up to two times to make $3 \mu \mathrm{m}$ thick porous platinum layers (Figure 6). $\mathrm{Li}^{+}$cations are used on all IPMC to obtain faster responses.

2.2. Excimer Laser Process. The electrodes on the tube and fiber are cut into four sections using an excimer laser process. The laser beam removes the plated surface in order to form four sections around the IPMC. These four sections drive the IPMC in 2.5 degrees of freedom. Figure 7 shows the micropump propulsion using tubular IPMC while Figure 8 represents one microleg. Both Figures are resulting from the laser process. This configuration around the IPMC should allow eight directions of motion labeled as $S_{1}$ to $S_{8}$. This technical process is also used to build up the BMR body and induction coil for the power supply.

2.3. Hardware Test Bench. In order to obtain the dynamic behavior of microlegs under electrical solicitation, a scanning laser vibrometer is used to measure the tip (free end) speed of displacement as a function of both frequency and the electrical current induced. The effect of microleg length on the first vibration mode is also inspected in a fluid.

The beam formed by the actuator is clamped at one end and is acting freely in $18 \mathrm{M} \Omega$ deionized water at room temperature (Figure 9). The laser beam goes through an optical window and reaches the microleg perpendicular to its neutral plane. Refraction of light has not been taken into account in the related measurements.

The applied voltage is a periodic PWM signal programmed as an arbitrary waveform generator-like model HP33120A. The waveform consists of a 1024-point signal where half the points form a positive PWM and the other half a negative one. Instantaneous speed was measured at the tip of the actuator and stored for all duty cycles used in the PWM signals of the waveform. The waveform has a $3 \mathrm{~V}$ peakto-peak and is applied at 10 and $20 \mathrm{~Hz}$.

The current is measured using two types of instruments. The first type is a current probe that measures the electromagnetic field around the cable (Tektronix P6042). The second type is a transimpedance amplifier (with gain set at 100 using the resistor) placed in serial with $\mathrm{H}$-Bridge MOSFET and IPMC. The isolation circuit using H-bridge MOSFET for the stimulus circuit is placed to drive opposite electrodes on IPMC microlegs. This circuit is used with the microcontroller parallel output port to drive all six microlegs and one vector control propulsion (this represents a total of 28 electrodes).

These measurements were done far away from the first resonance of the mechanical system: a modal analysis of the microleg using a randomly applied voltage and scanning capability of the vibrometer revealed an initial natural frequency of $180 \mathrm{~Hz}$. This frequency takes into account the coupling between the fluid and the structure.

\section{Microleg Model}

Our previous paper demonstrates three-dimensional microleg movements with mathematical equations and simulation for the actual electrode configuration [10, 11]. Two voltage amplitudes were simulated. This section presents all results for the frequency response and includes theoretical explanations and an analysis of each result.

3.1. Analog Electrical Stimulus. Microlegs will be driven by a voltage-controlled CMOS technology. It is important to know these stimulation effects on the current consumption at any frequency.

The current transient step response shows a major current peak that decreases rapidly (Figure 3). A negative peak is also produced when the square wave stimulus return to zero. This 


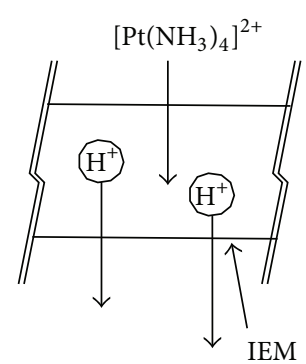

(a)

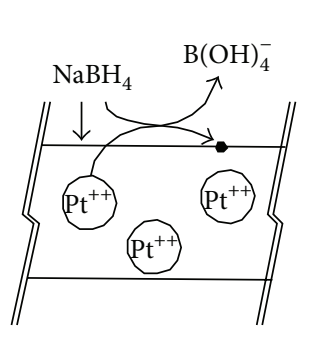

(b)

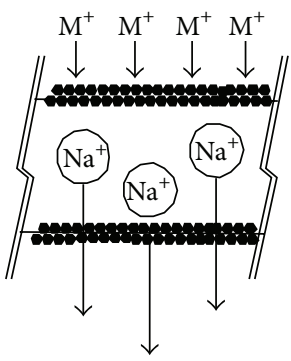

(c)

FIGURE 5: Chemical process of Nafion plating with platinum [20].

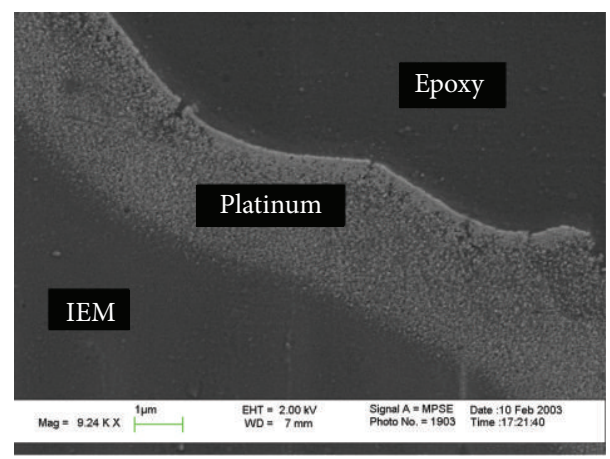

Figure 6: IPMC cross section.

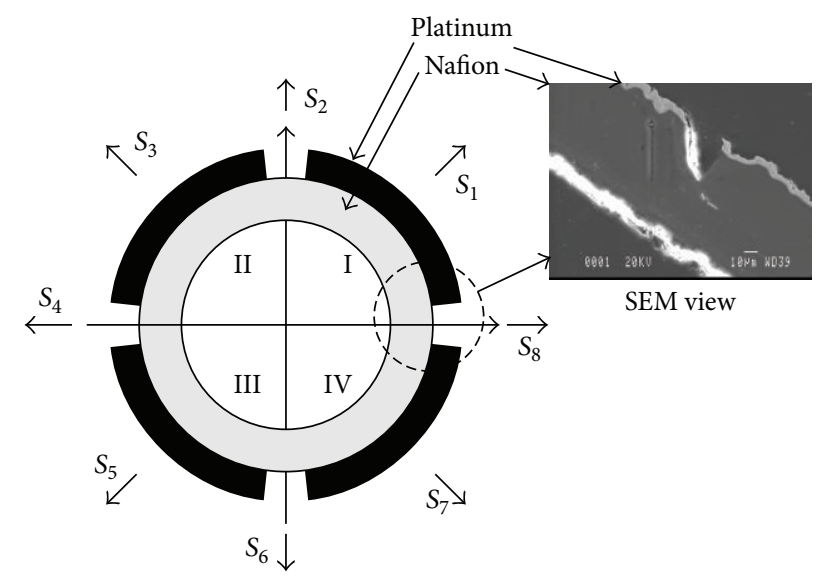

FIGURE 7: Tubular IPMC for the micropump propulsion.

phenomenon is explained by a serial resistance-capacitance $\left(2 R_{s}+C_{\mathrm{dl}}\right)$ network introduced via the electrode-electrolyte interface (Figure 10).

Capacitance is due to double-layer capacitance $C_{\mathrm{dl}}$. If the voltage is increased, $C_{\mathrm{dl}}$ increases until the electron transfer occurs at the electrode/solution interface. The continuous current remains in steady state, which shows a nonlinear resistive element $R_{F}$ through the IPMC parallel to $R C$ network [21]. This reaction was analyzed previously for different IPMC and cations types [22]. Normally, electroneutrality is maintained across the interphase, but it may be leaky, allowing some faradic current to flow across it, explained

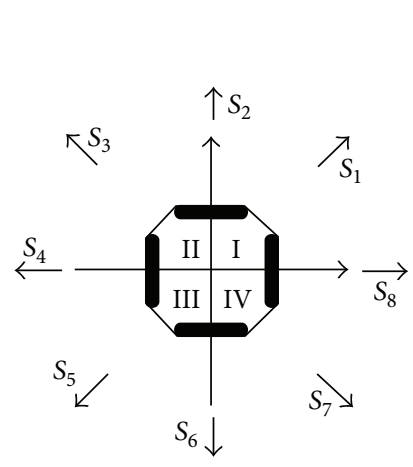

(a)

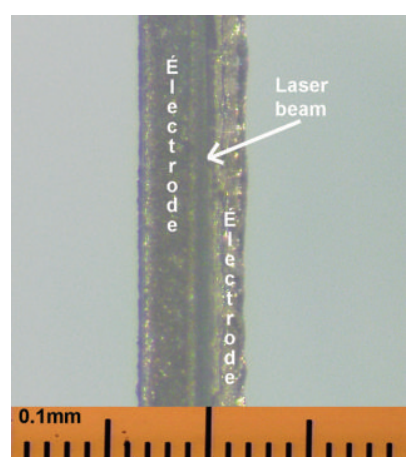

(b)
FIGURE 8: Square section of the microleg.

by faradic resistance $R_{F}$. $R_{F}$ may behave as simple ohmic resistance, but it also can be a hyperbolic or an exponential function of potential, depending on the range of overpotential considered. $C_{\mathrm{dl}}$ also is a complex function of potential, although it may not vary as much as $R_{F}$ [23].

Analysis of microleg electromechanical characteristics does not need to include an analysis of Warburg impedance and absorbing pseudocapacitance due to the limitation of mass transport by the diffusion and transfer process of charge. However, it is important to note that Warburg impedance adds a constant phase of $-90^{\circ}$ between current and voltage, which may influence transient or frequency analysis as seen in Figure 11(b). Only $C_{\mathrm{dl}}$ is treated as pure capacitance, in the physical sense.

Electrode surface resistance, $R_{S S}$, can be represented and explained by low conductivity, insufficient electrode thickness, microscopic cracks and the heterogeneous deposition of metal particles. The cyclic deformations in traction and contraction during cyclic movement of the IPMC can cause fatigue and an increase in this resistance $[24,25]$. The network $R_{F} / /\left(2 R_{S}+C_{\mathrm{dl}}\right)$ is an approximation of the proposed model.

In steady state, two regions have been defined for the microleg: the linear zone below the decomposition voltage (sum of metal overpotential used as the electrodes and standard equilibrium potential or standard electrode potential) and nonlinear exponential zone above decomposition voltage (Figure 12).

The frequency response shows interesting particularity. Measurements are taken using the sinusoid $2 \mathrm{~V}$ peak-to-peak. 


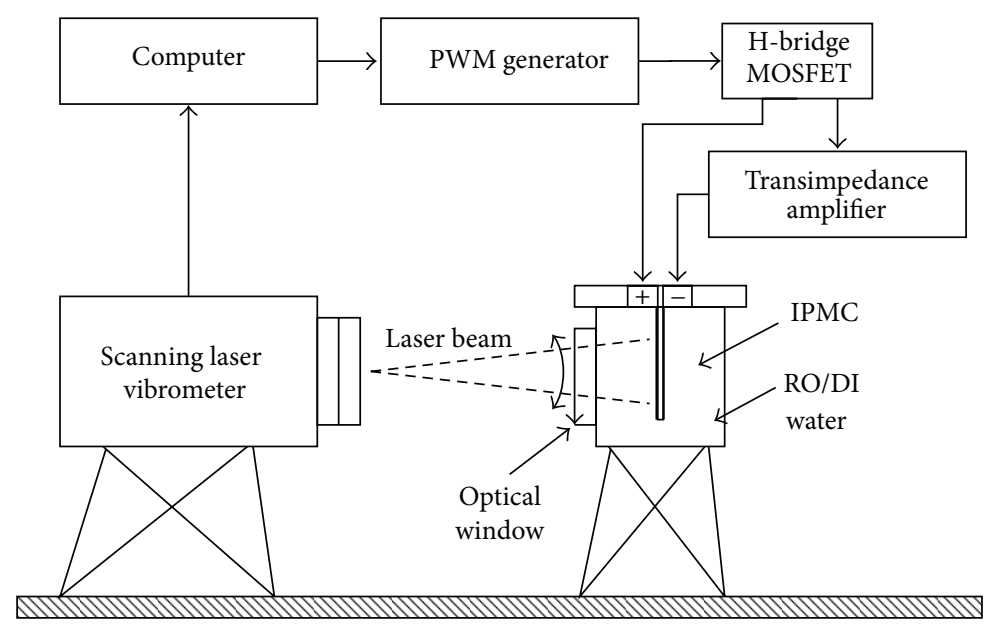

FIGURE 9: Experimental setup for microleg speed measurement.

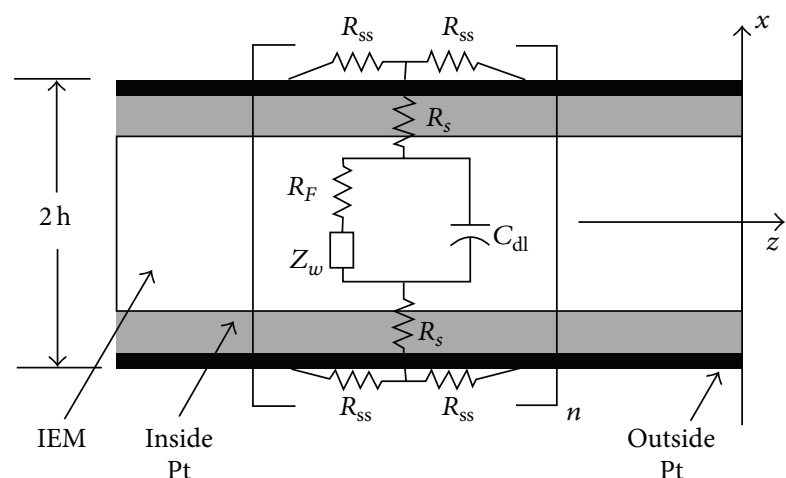

Figure 10: IPMC electrical model [24].

The current amplitude response has a gain of $-10 \mathrm{~dB} /$ decade between 5 and $100 \mathrm{~Hz}$ and cut-off frequency near $45 \mathrm{~Hz}$. As well, linear characteristics are associated with the phase frequency response.

This characteristic shows that current is out of phase from voltages between $-100^{\circ}$ and $-180^{\circ}$. It can be explained by the presence of absorbing pseudocapacitance and Warburg impedance.

3.2. Mechanical Displacement Analysis with PWM. Analysis carried out until now does not make it possible to control the IPMC bending angle using CMOS technology. RISC microcontroller architecture does not support analog output at the 8-bit parallel port [26]. Adding a digital-to-analog converter (D/A) is a cumbersome solution for MEMS and/or microproduction. Although the IPMC microleg can be modeled using a $R / / R C$ network, the use of PWM modulation can adjust the quadratic mean of the electric tension $V_{\mathrm{RMS}}$. Next, the bending angle can be controlled if a relationship exists between the IPMC bending angle and PWM duty cycle.

However, the first step is to check whether a certain dependence or correlation exists between the duration of PWM impulse and microleg displacement. The scanning laser vibrometer cannot directly measure displacement, because it can only analyze dynamic structure. Therefore, it is necessary to study the dynamic behavior in a cantilevered beam configuration, that is, microleg tip displacement rate function of the PWM duty cycle. The waveform generator provided with scanning laser vibrometer requires programming the desired signal.

For the experiments, the IPMC is supplied periodically on each one of its electrodes. This periodic signal vibrates the structure within two degrees of freedom. This implies the periodic generation of the standardized signal. Parameter adjustment (frequency of the signal and the voltage amplitude) on the standardized signal makes it possible to obtain excitation signals used for the experiments. The signal is adjusted with a value of a 6-volt peak-to-peak for two frequencies: 10 and $20 \mathrm{~Hz}$.

Figure 13 shows the tip microleg displacement rate in a cantilevered beam configuration for $10 \mathrm{~Hz}$ and $20 \mathrm{~Hz}$ for the odd PWM duty cycle. Figure 14 shows a mean maximum speed for each PWM duty cycle over the 30 measurements taken from data as found in Figure 13. Experimental results may be expressed as linear characteristics. The next analysis shows the degree of correlation between the mean maximum speed movement and PWM duty cycle.

The correlation coefficient $r$ calculation provides an estimate of the correlation degree between two random variables of even population. The value obtained for $r$ must be checked to ensure that it does not differ significantly from 0 , which probably indicates a linear absence of the correlation between the variables observed. A significance threshold of 0.05 and a population of ten (10) samples for the mean maximum are used for the analysis. The variation of vibration function of frequency is distributed according to Student Law (bilateral case) with 8 degrees of freedom. Since the value of $r^{2}$ is high (0.982 and 0.986), as shown in Figure 14, the straight line adjustment at the experimental points is a good quality. It is obvious that this study cannot show cause-to-effect between the two variables. However, predictable actuator mechanical and physical behaviors make it possible to validate that there is a cause-to-effect between 


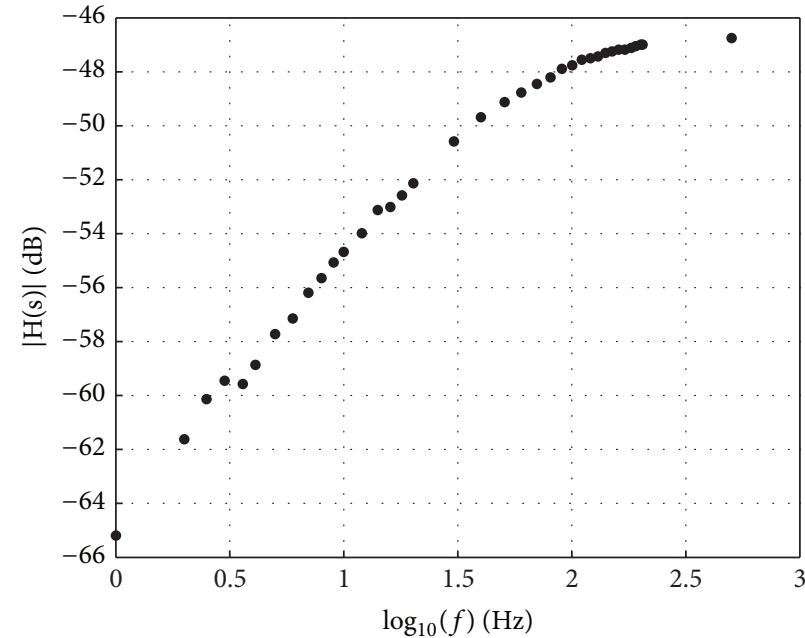

(a)

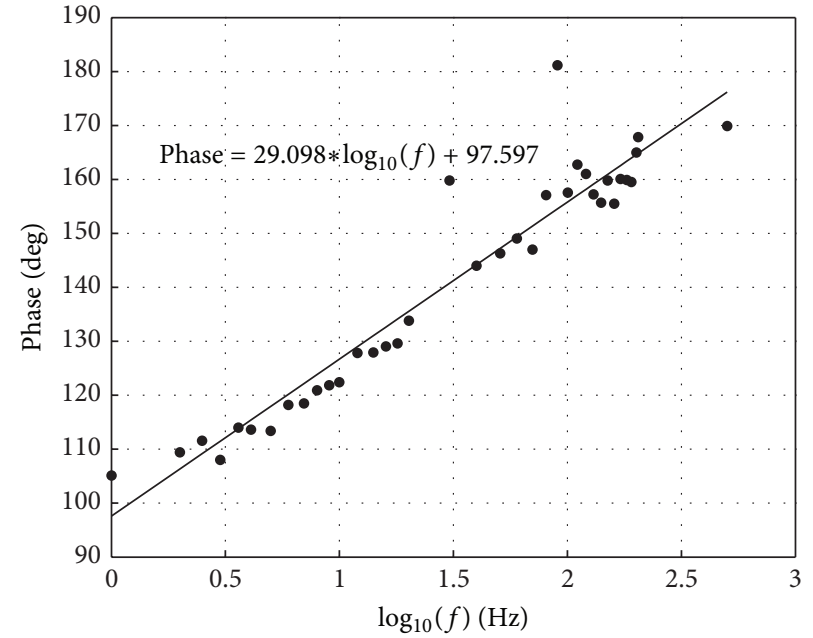

(b)

FIGURE 11: Current amplitude (a) and phase (b) response ratio of voltage amplitude function of frequency.

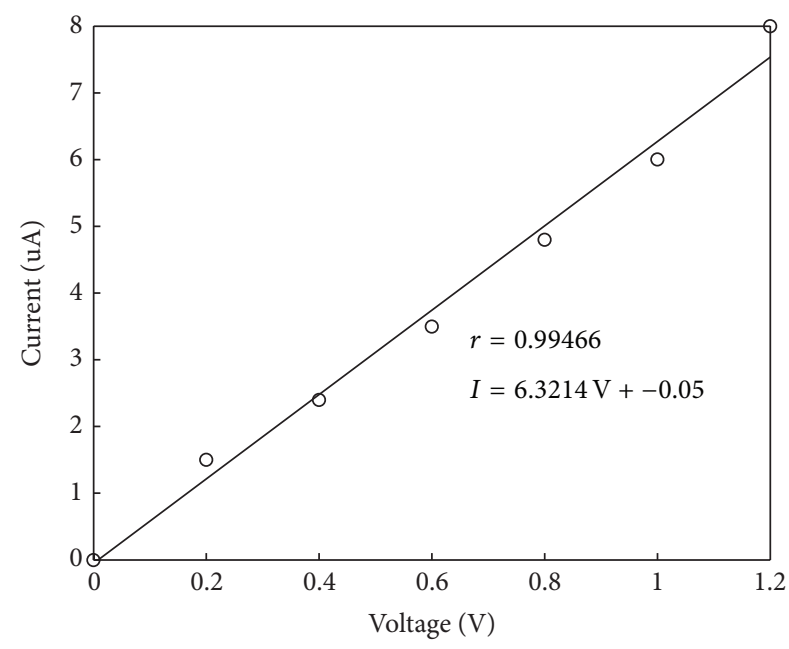

(a)

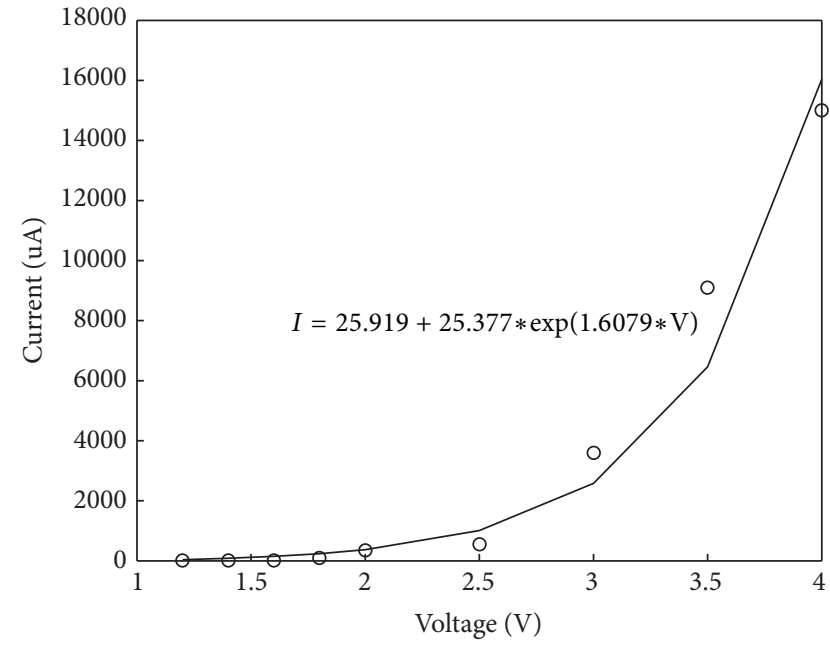

(b)

FIGURE 12: Steady state current function of voltage in (a) linear and (b) nonlinear regions.

the two independent variables: the variations of one variable involve the variations of the other without an unspecified attribution of an external common cause variation.

3.3. Frequency Analysis Using FFT. Starting from these results, it is possible to investigate more IPMC properties in the frequency domain. A structure of this type has particular modes of resonance that can be used in the propulsion of the robot as a strategy for reaching optimal motion. It was shown that IPMC dimensions vary its natural frequency: the natural frequency decreases when its length increases in a similar way to a cantilever beam [27].

The distributed systems have an infinite number of degrees of freedom and natural frequencies. Each natural frequency has a single mode, which is known to be like a normal function. A transitory vibration forced or in a steady operation will generally excite several or all the frequencies and modes in combination. The clear response in a point can be expressed in their terms according to the superposition principle. The first mode is that associated with the weakest frequency [28]. The use of the first vibration mode will be probably advantageous for moving the robot at its optimal speed in terms of current consumption versus velocity. Thus, it is necessary to theoretically and experimentally analyze the IPMC first modes in the cantilever beam configuration.

In the case of the IPMC microleg, free end tip mass is negligible. Next, the resolution of theoretical equation provides all the proper frequency $f_{n}$ using [29]

$$
f_{n}=\frac{1}{2 \pi} \frac{k_{n}^{2}}{L^{2}} \sqrt{\frac{E I}{\rho S}}, \quad S=2 l h,
$$

where $L$ is the IPMC length, $l h$ product is the IPMC cross section $(0.2 \mathrm{~mm} \times 0.2 \mathrm{~mm}), I$ is the inertia, and $E$ the Young 


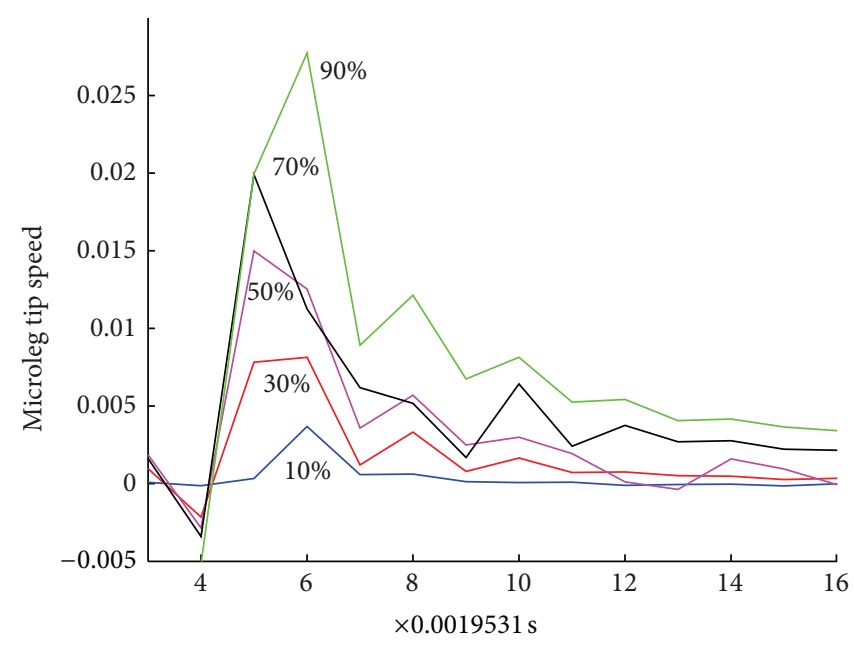

$10 \mathrm{~Hz}$ PWM

Duty cycle (\%)

$10 \%$
$-30 \%$

$-50 \%$

(a)

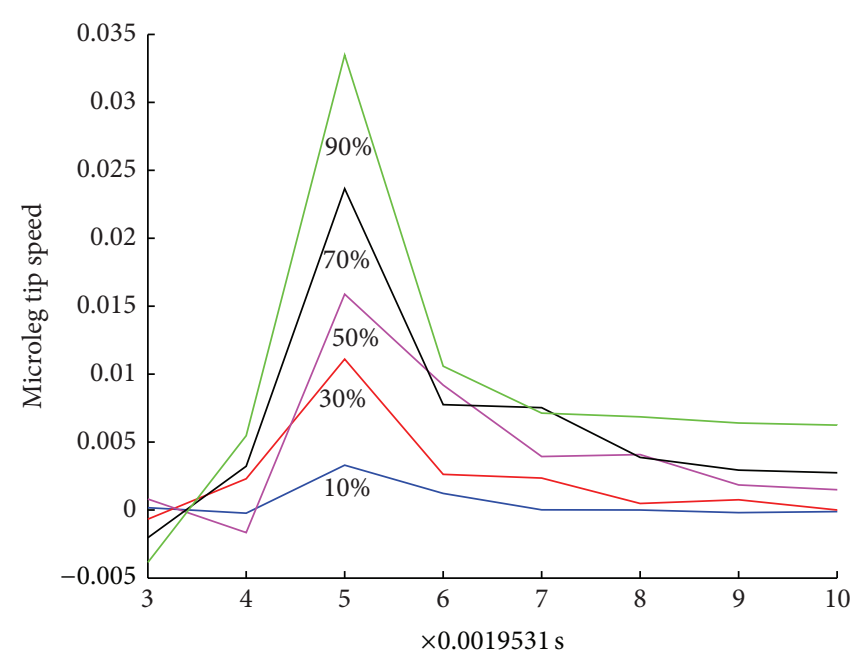

$20 \mathrm{~Hz}$ PWM

Duty cycle (\%)

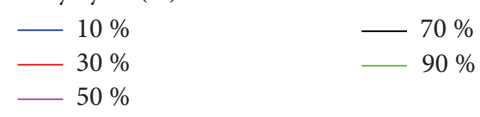

(b)

FIGURE 13: Tip microleg displacement rate in function of multiple PWM duty cycle at PWM period of (a) $10 \mathrm{~Hz}$ and (b) $20 \mathrm{~Hz}$.

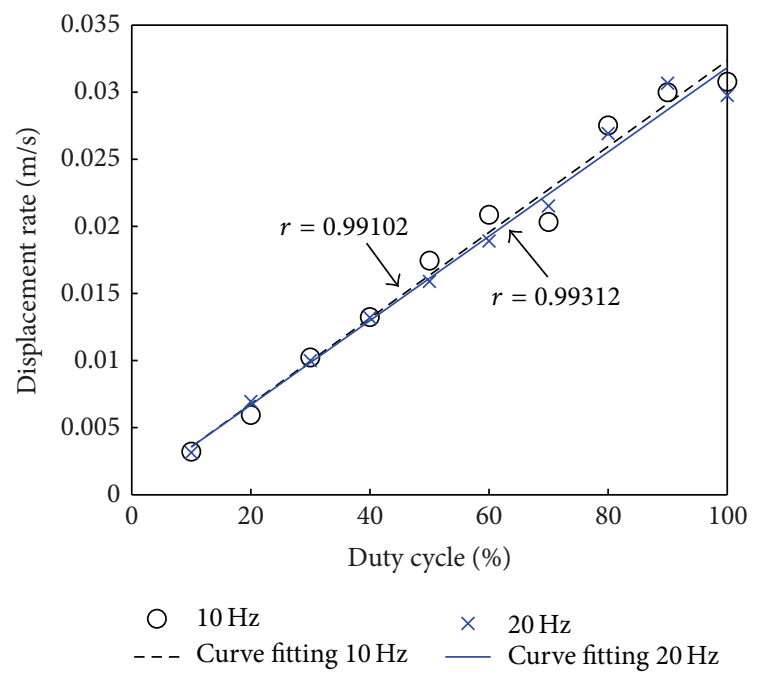

FIGURE 14: Mean of maximum tip speed as a function of PWM duty cycle.

modulus. $k_{n}$ comes from the resolution of characteristic equation:

$$
1+\cos \left(k_{n}\right) \cosh \left(k_{n}\right)=0 .
$$

However, this equation is not valid within the water environment. It is necessary to consider the behavior of a fluid-structure coupled system. In general, it should be checked whether the fluid is incompressible. In this case, the effect of fluid is then purely inertial. Using the representation of added mass makes, it possible to find a simple formula to evaluate the natural frequency of the coupled system. When the structure vibrates in water, it induces water acceleration, producing an additional force on the structure, in addition to the force of trail fluidic-dynamics. This added force can be suitably modeled as the product of hypothetical water mass and structure acceleration. This implies that the structure in water vibrates at a natural frequency weaker than the frequency in a vacuum. By replacing inertial $I$ of a circle of radius $a$ and its surface $S$ in (1) of the eigen frequencies $f_{n}$, the result is given by

$$
\begin{gathered}
I=\frac{\pi(2 a)^{4}}{64}=\frac{\pi(a)^{4}}{4}, \\
S=\pi a^{2}, \\
f_{n}=\frac{1}{2 \pi} \frac{a k_{n}^{2}}{2 L^{2}} \sqrt{\frac{E}{\rho}} .
\end{gathered}
$$

The equation describing a vibrating beam in water should comprise both the structure density of the beam described in (1) and that of water, which corresponds to a coupled system in density. The coefficient of added mass $C_{m}$ defined as the ratio of the added mass to that of water moved by the cylinder makes it possible to couple the structure with water according to

$$
f_{n}=\frac{1}{2 \pi} \frac{a k_{n}^{2}}{2 L^{2}} \sqrt{\frac{E}{\left(\rho_{c}+C_{m} \rho_{w}\right)}} .
$$

A simulation of (3) and (4) is shown in Figure 15. To prove this theory, modal analysis was carried out with the 


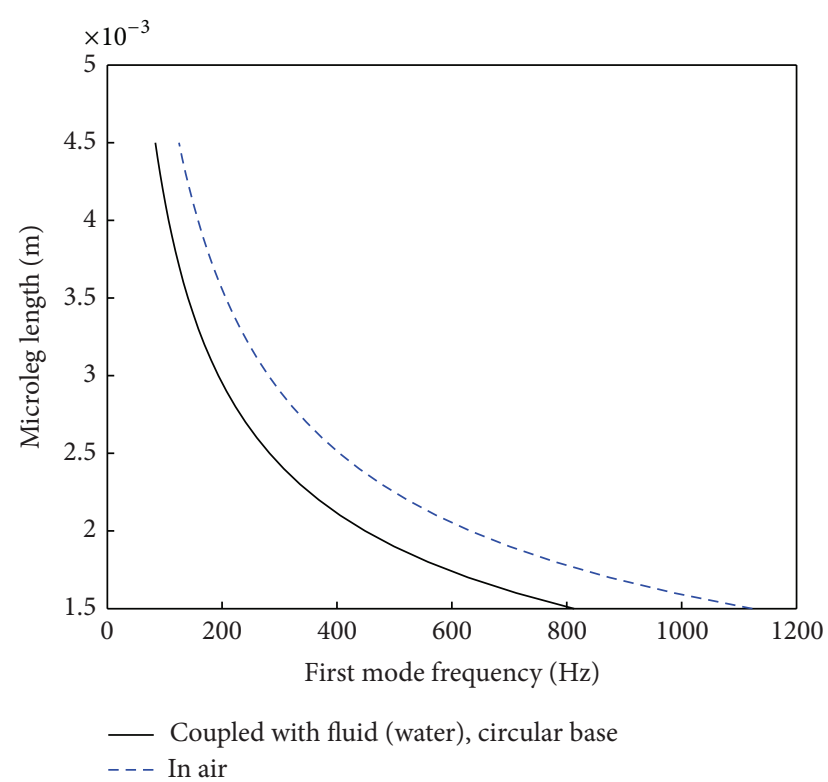

FIGURE 15: Simulation of the microlegs first mode frequency functions of its length.

scanning laser vibrometer using a pseudorandom excitation voltage (Gaussian white noise) of $6 \mathrm{~V}$ peak-to-peak. This analysis makes it possible to find the first mode vibrating frequency $F_{r 1}$ at about $180 \mathrm{~Hz}$ for a 4 -millimeter microleg length (Figure 16). This frequency takes into account the coupling between heavy fluid (deionized water) and the structure. A digital simulation is used to find a frequency close to that found with the laser. Moreover, it is interesting to compare the tension/current cut-off frequency (about $45 \mathrm{~Hz}$ from Figure 11(a)) with the maximum frequency $F_{\operatorname{MAX}}$ at about $50 \mathrm{~Hz}$. Note that frequency response fluctuates according to the applied voltage and according to resistance $R_{F}$ and capacitance $C_{\mathrm{dl}}$ values.

\section{Water Electrolysis}

Many other IEM membranes can be used for this experiment, such as Flemion or Aciplex. Flemion-Au IPMC, with its dendrite inside ionomer, is known to have a higher ion exchange capacity, higher surface electrical conductivity, higher hydration capacity, and higher longitudinal rigidity than NafionPt. These conclusions provide Flemion-Au with a better curvature angle for the same applied voltage without relaxation [30]. Gold can be also envisaged given its advantages: stable in acid, ductile, good electrical conductor, and less reactive in electrochemical reactions [31,32]. Gold has also an overpotential higher than Pt. It allows using higher voltages to produce bending curvature without water electrolysis.

This section presents different ways to avoid water electrolysis. Currently, cation type and electrode metal have demonstrated some useful characteristics for avoiding backrelaxation when stimulus is applied and for avoiding water electrolysis. First, an overview of cation and electrode characteristics is presented. Second, a new way to avoid electrolysis

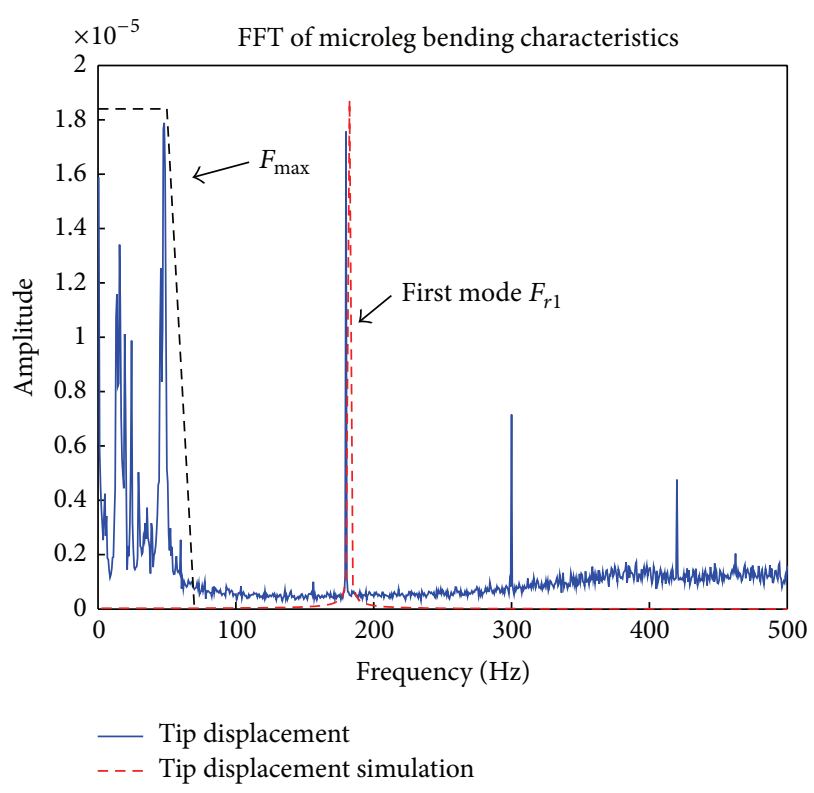

FIGURE 16: Modal analysis with laser vibrometer in frequency with FFT.

is introduced with signal waveform and frequency applied as the stimulus.

4.1. Cation Type. Cations can be divided into three classes: $A, B$, and $C$. These classes are categorized by water ionic conductivity and water state inside IEM. The $A$ class contains small hydrophilic alkali and some alkaline-earth metal $\left(\mathrm{Li}^{+}, \mathrm{Cu}^{2+}, \mathrm{Na}^{+}, \mathrm{K}^{+}, \mathrm{Rb}^{+}\right.$, and $\left.\mathrm{Cs}^{+}\right)$. The $B$ class is formed by hydrophobic alkyl ammonium ions $\left(\left(\mathrm{CH}_{3}\right)_{4} \mathrm{~N}^{+}\right.$, $\left(\mathrm{C}_{2} \mathrm{H}_{5}\right)_{4} \mathrm{~N}^{+}, \mathrm{NH}_{4-m}\left(\mathrm{CH}_{3}\right)_{m}{ }^{+}$, and $\left.\mathrm{NH}_{4-m}\left(\mathrm{C}_{2} \mathrm{H}_{5}\right)_{m}{ }^{+}\right)$. Lastly, class $\mathrm{C}$ includes the large hydrophobic cations, such as TBA (tetrabutylammonium $^{+}$) and TPrA (tetrapropylammonium) [33].

Water displacement volume inside polymers causes contractions and dilations, which involves asymmetrical pressure distribution. Thus, internal stresses produce temporary polymer deformation. For small cations, like $\mathrm{Li}^{+}$, the displacement response is fast. However, if tension is maintained, the polymer will not keep its shape with a Nafion-Pt composite [22]. From another perspective, for bulkier cations, like tetran-butylammonium ${ }^{+}$(TBA), the response is slower, but there will be a little relaxing. The relaxation time depends on the water leakage in the polymer interstices.

Relaxation probably occurs since the cations transport more water molecules than there should be in an equilibrium state. Relaxation will finish when equilibrium has been reached between the osmotic water pressure (dilution of the ions types) induced by electrical voltage, interfacial polymersolvent, and polymer internal elastic energy (limitation of polymer chain elongation) [34]. No further relaxation is observed with TBA cation type. It can be caused by the possibility that the cations block the channels and prevent water leakage or by the possibility that the concentration of cations requires more water than it moved. No relaxation 
occurs with Flemion-Au IPMC for all cation types. Instead, the displacement rate drops without moving back [22].

If ammonium size increases, IPMC displacement rate will go down, because of the slower molecule mobility inside the ionomer. However, the IPMC curving angle will be more pronounced, and relaxation by water leakage will decrease gradually. Moreover, ammonium's slower mobility eliminates electrochemical reactions like water electrolysis [35].

4.2. Electrode Characteristics. Electrode polarization, by electrical current, should alter half-cell potential. Half-cell variation (difference between observed half-cell potential and equilibrium zero current half-cell potential) is known as overpotential. The element chosen for electrode composition is directly associated with IPMC performance such as ductility and electrical conductivity. Electrodes must be also stable in electrochemical reactions like noble metals. Of course, overpotential should be very large for a higher decomposition potential. This section explains the effects of overpotential.

Electrodes made with noble metal, such as platinum and gold, have a behavior similar to perfectly polarizable electrodes. Both platinum and palladium have extremely small overpotentials for hydrogen evolution. Gold has a significantly larger overpotential. As well, gold does not appreciably absorb hydrogen, and this factor together with its larger overpotential for hydrogen evolution makes gold the metal of choice [21]. Such a relatively inert electrode is difficult to oxidize and dissolve. The best reaction for IPMC is characterized by a displacement current (no actual charge crosses the electrode-electrolyte interface) and the electrode behaves as though it were a capacitor. In this case, the majority of the overpotential is a result of concentration overpotential (ion concentration variation at the interface) [36].

Frequency and waveform have a direct influence on ion mobility inside IEM. The next section explains frequency and waveform influence on the displacement current at the interface to approach IPMC behavior as a capacitor without a resistive element.

4.3. Frequency and Waveform. Visual observations of microleg displacement show that the movement is greater in the nonlinear region. This result is not interesting for many biomedical applications. However, it is possible to drive the microleg with a charge-balanced waveform.

Control of the charge density and charge balance are essential for avoiding electrolysis and to provide a chemically reversible reaction (or safe stimulation). Waveforms can be symmetrical or asymmetrical. Symmetrical refers to biphasic stimuli in which the magnitude of the current density in the first pulse of the biphasic pair is the same as in the second pulse. The use of biphasic stimulus waveforms with zero net charge flow does not guarantee that toxic electrochemical reaction products will not be produced [37].

Considering the IPMC electrical model, a minimum frequency is necessary for eliminating water electrolysis. The electrochemical technique of cyclic voltammetry can delineate an operational potential window between hydrogen and oxygen evolution. This technique is applied to find minimum frequency of symmetrical stimuli because water electrolysis decreases thermodynamic efficiency and consumes power.

Figure 17 shows three hysteresis curves for the frequency 10, 30, and $180 \mathrm{~Hz}$. Signal amplitude applied to system is $5 \mathrm{~V}$ peak-to-peak in order to produce water electrolysis. To read current amplitude, divide $\Delta Y$, on this figure, by 100 , which is the transimpedance amplifier gain. Water electrolysis is shown by concave form at two hysteresis extremity. It is clear that increasing frequency decreases power consumption and eliminates water electrolysis.

A study could be carried out to characterize the mechanical reactions during stimulation with a current source instead of a voltage source. The use of a current source would make it possible to control the quantity of charge flow injected by varying amplitude and/or the width of the pulse phase with any IPMC impedance. Maximum charge density being able to be injected is according to the surface of the electrodes.

\section{Locomotion Model and Control}

A very special design will be developed and explained to allow BMR to move in all directions within the constraints of the actuators and the eight-bit parallel port of the microcontroller [26]. In this vein, the first part of this section is devoted to the study of the dynamics of an insect gait. This study highlights the main parameters to be considered in order to operate the microleg. Among other things, the types of locomotion are covered. The type of locomotion is important because it depends on the speed of the insect and hardware constraints of the microcontroller. The analysis of the movement of the leg and the movement of an insect with six legs, like ants, leads the design of the control system. It will also be necessary to understand the functioning of the physiognomic locomotion in the central nervous system for a robust adaptive algorithm.

5.1. Insect Locomotion. At this time, many works have studied the decentralized and central control systems involved in locomotion. The combination of behavioral and electrophysiological experimental data shows that locomotion is the result of both central and decentralized mechanisms [38]. This implied that each leg has an autonomous sensitive element controlled independently.

The RISC microcontroller architecture used in this application has only one eight-bit parallel port [26]. This strong internal constraint only lets us use the central control. In fact, decentralized locomotion control would require a 28 -output bits on one parallel port ( 6 legs $\times 4$ electrodes per leg, plus the micropump using thrust vectoring control) from the microcontroller to drive each BMR leg independently without sensitive input and reflex. Next, this paper is based essentially on central systems. With this bus width, a simple neural network architecture called Walknet, a biologically inspired network for controlling six-legged walking, can be used from a cellular nonlinear approach $[39,40]$. The central command developed here coordinates BMR movements with a higher stereotypy and less adaptivity. A feedback loop acts as a reflex to adjust the leg displacement. This reflex can move one leg to another position to avoid or to climb over an obstacle. 


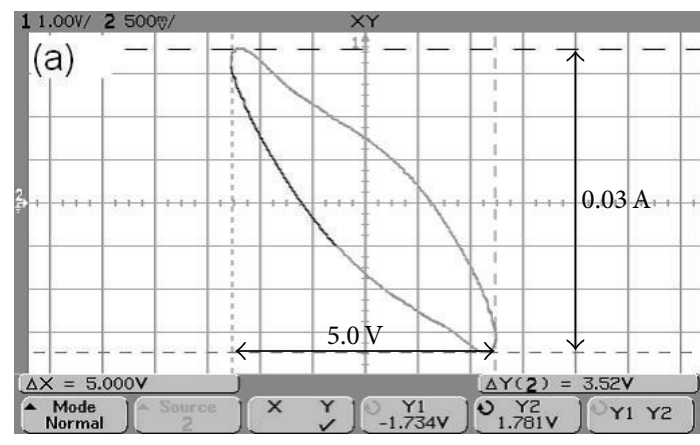

(a)

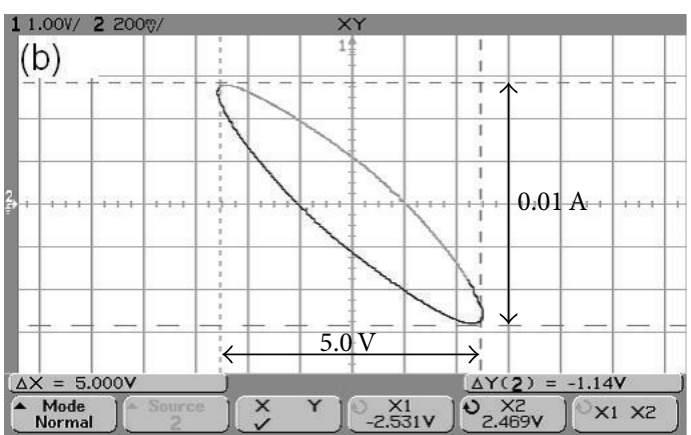

(b)

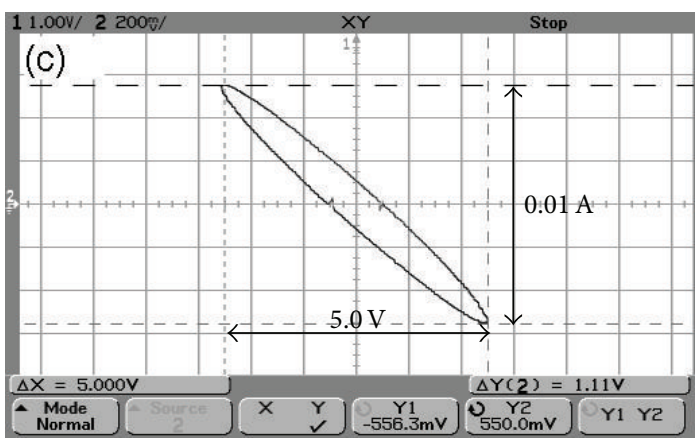

(c)

FiguRE 17: Hysteresis of current response for (a) $10 \mathrm{~Hz}$, (b) $30 \mathrm{~Hz}$, and (c) $180 \mathrm{~Hz}$ sinusoid voltage for the same voltage amplitude applied.

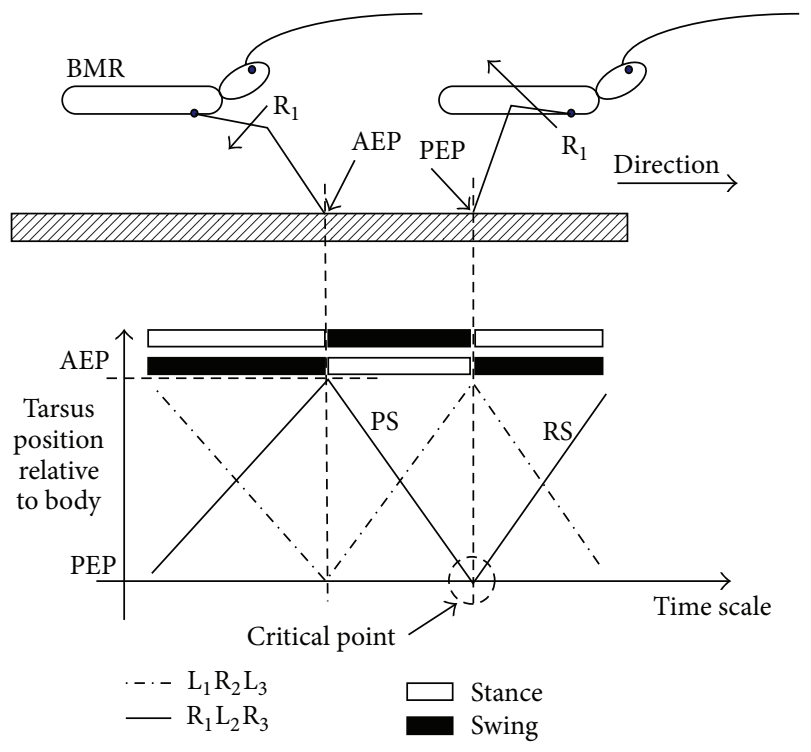

FIGURE 18: The cyclic movement of a leg and the tripod (fastest gait) walking stepping pattern.

5.2. Hexapod Insect Walking. The cyclic movement of a walking leg consists of two parts (shown in Figure 18), the power stroke (PS, also stance or support phase that propels the body) and the return stroke (RS, also swing or recovery phase).

The anterior transition point (transition from swing to stance) is called the anterior extreme position (AEP), and

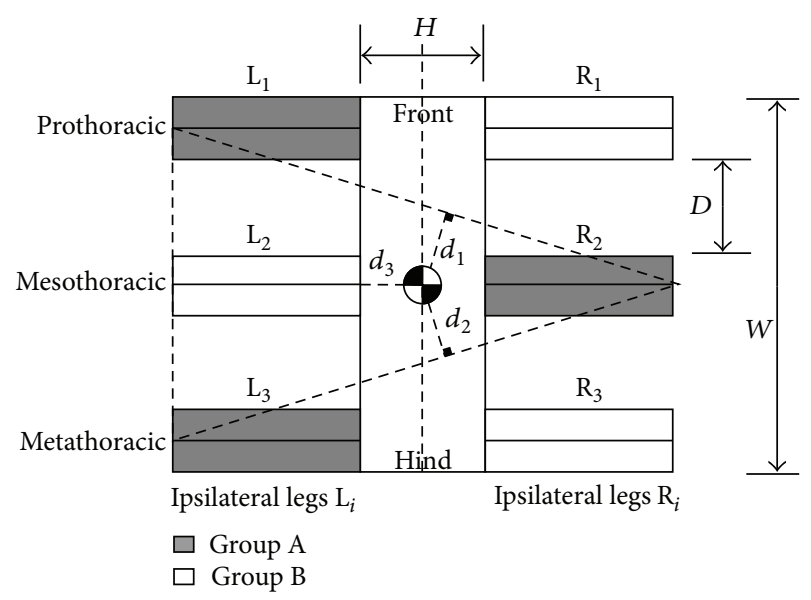

FIgURE 19: Top view of microrobot. Two legs groups (A, B) must be used. The tripod of support is represented by the dash triangle.

the posterior transition point is called the posterior extreme position (PEP). The most critical point to maintain the robot's stability is the transition from PS to RS [41]. Next, an interesting location for the feedback sensor could be used to detect PEP movement.

Two conditions must be met to maintain the robot's stability during all locomotion phases. The center of gravity of the body must lie within the triangle of support as shown in Figure 19. As well, three feets must always be on the ground simultaneously. The body is supported alternatively, for equal 


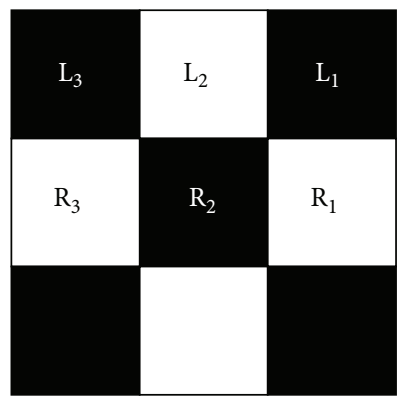

$\square$ Stance
$\square$ Swing

(a)

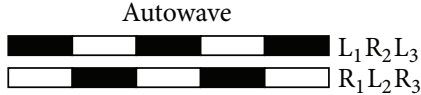

Stance

Swing

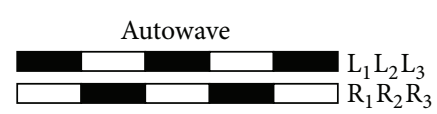

$\square$ Stance

(b)

(c)

FIGURE 20: (a) Checkerboard Turing pattern corresponding to tripod gait locomotion. (b) Insect fast-gait pattern. (c) Insect swimming pattern.

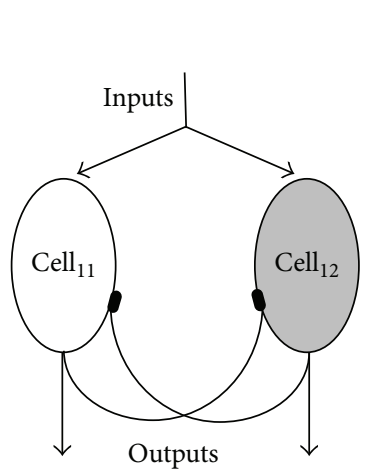

(a)

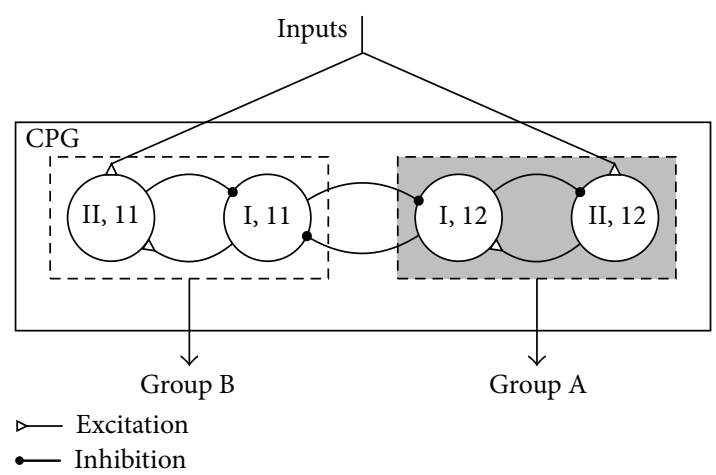

(b)

Figure 21: (a) Reciprocal inhibition network consisting of two similar neurons (or two populations of neurons). (b) CPG CNN design with reciprocal inhibition network.

periods of time, by one of the two groups of legs (tripod gait): A $\left(\mathrm{L}_{1} \mathrm{R}_{2} \mathrm{~L}_{3}\right)$ or $\mathrm{B}\left(\mathrm{R}_{1} \mathrm{~L}_{2} \mathrm{R}_{3}\right)$.

The stability margin is defined as the shortest distance $\left(d_{1}\right.$, $d_{2}$, and $d_{3}$ ) from the center of mass to the edge of the tripod of support. Many insects closely conform to this pattern when moving quite rapidly $[42,43]$. Two main strategies are known to allow the insect to turn: increasing step frequency or step length of legs on one side of the body versus the other [44].

5.3. Central-Pattern Generator Paradigm. The central nervous system (CNS) must produce specific patterns of motor neuron impulses during coordinate locomotion or movement. The central hypothesis purports that rhythmic movements or basic motor programs in walking are driven by the central-pattern generator (CPG) located in the CNS. The
CPG includes subnetworks of command neurons (CNs) and local pattern generating neurons (LPGNs). The neurons of LPGN are called motoneurons (flexors and extensors neurons) $[45,46]$. The impulse in nerve membrane is modeled using the nonlinear reaction-diffusion partial differential equation (RD-PDE), which can be represented by autowaves with cellular neural networks (CNN). Its capacity to generate plateau potentials or oscillations is a key issue for rhythm generation, but also for driving the transition among various types of locomotion like walking, running, and swimming [47].

Taking into consideration the biological aspects of locomotion, reaction-diffusion CNN (RD-CNN) is made up of two parts: one is devoted to generating autowaves (Figures 20(b) and 20(c)) and the other is responsible for specific 


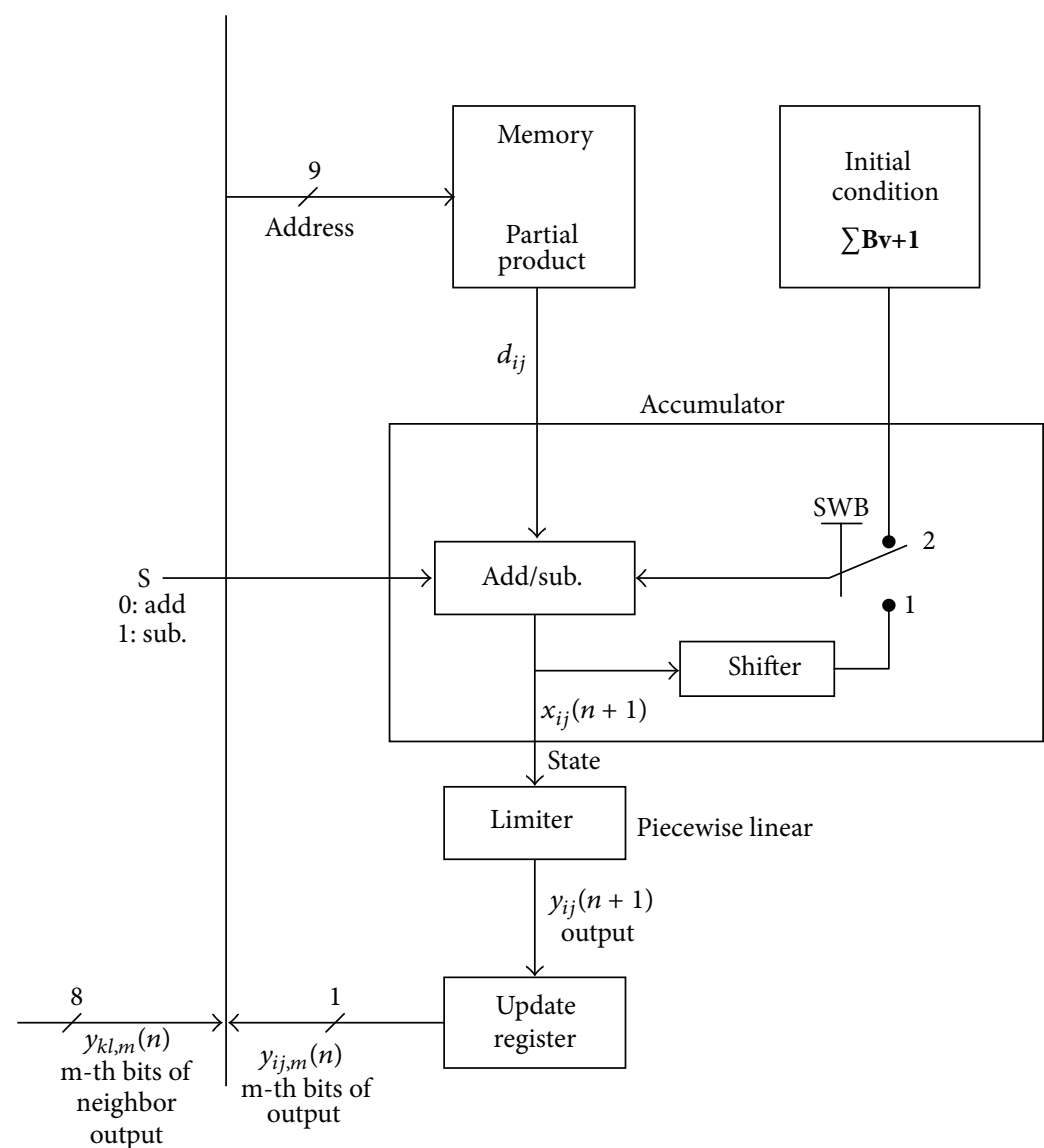

(a)

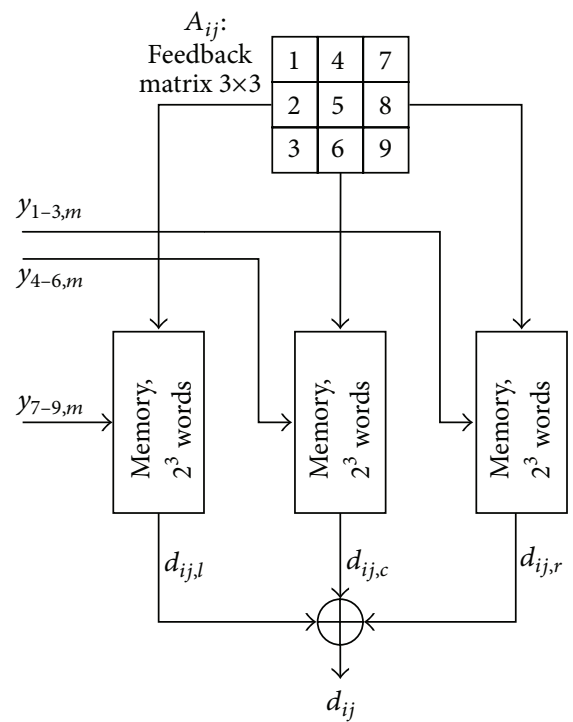

(b)

FIGURE 22: (a) Block diagram of DTCNN cell. (b) Memory partitioning scheme.

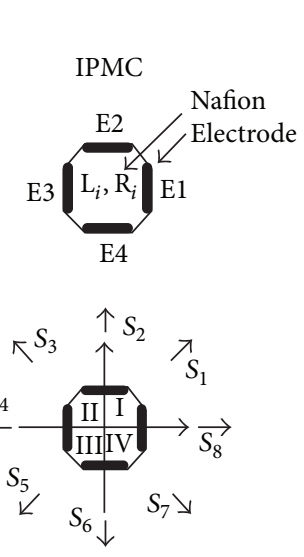

(a)

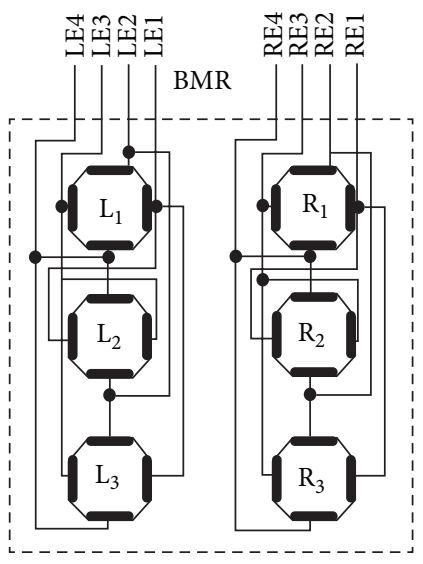

(b)
FIGURE 23: (a) Leg cross section with four electrodes in Cartesian coordinates. The states $S_{i}$ generate bending moments and the associated direction movement. (b) BMR body hardware.

types of locomotion obtained with a stable checkerboard Turing pattern configuration (Figure 20(a)) [48]. Multitemplate approach (MTA) is an extension of the model used in RDCNN-based hexapod robot. CPG implementation is based on the principle that the same group of neural cells can be reorganized by changing the synaptic connection [49].

For implementation purposes, CNN can be classified as continuous-time (CT) [50] and discrete-time (DT) models [51]. CNN is composed of basic nonlinear dynamic circuits known as a cell, where each unit is connected only as a set of adjacent cell neighbors to form an array $M \times N$. In this way, the $3 \times 3$ checkerboard Turing pattern needs a $1-$ neighborhood (Figure 22(b)). The CNN structure can form in any dimensions, but, in our application, we need only twodimensional arrays to reproduce autowaves for the tripod locomotion pattern.

An analogically programmable CNN in CMOS technology is reported with MOS transistor. This implementation is based on operational transconductance differential amplifier (OTA) stage. The cell resistor is approximated by the MOS transistor and its current-voltage relationship is nonlinear [52]. Moreover, digital architecture for the discrete-time $\mathrm{CNN}$ based on the combination of bit-serial computation of distributed arithmetic (DA) is presented. The system using DA has some advantages over all others: small memory size, shared memory contents, and reduced bus width. It can be implemented on an FPGA chip for reconfigurability, and a digital CMOS VLSI chip for high integration. The digital approach has the advantage of simplicity and expandability 


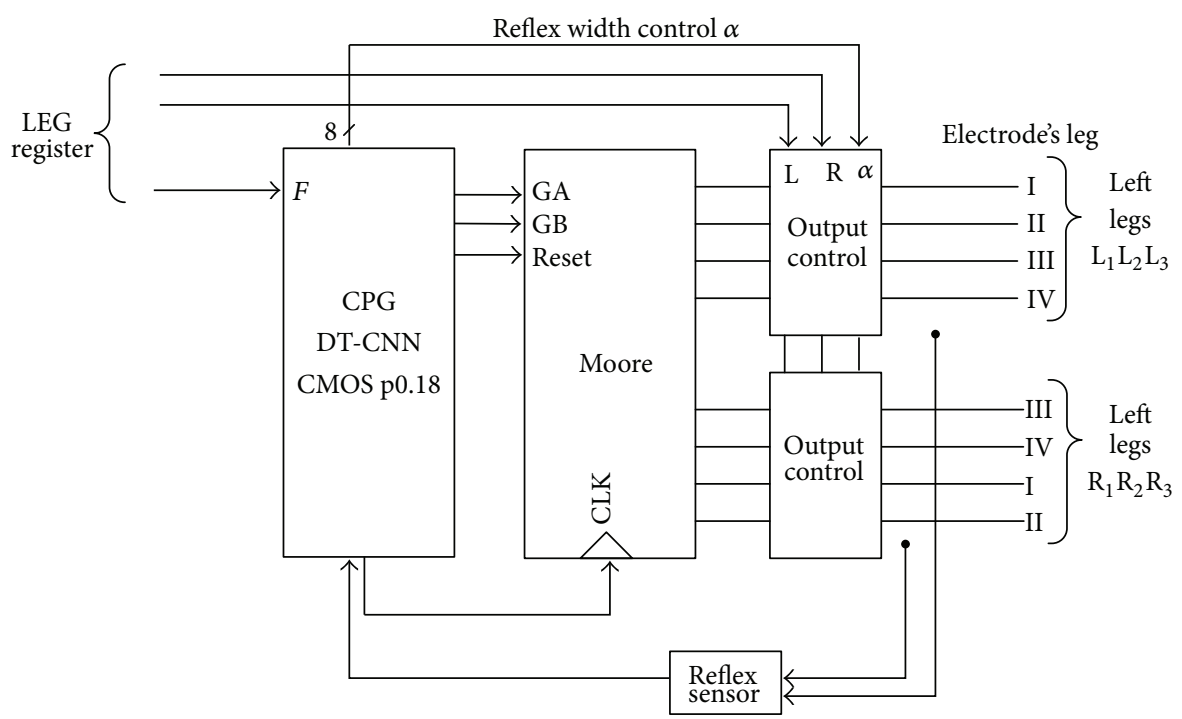

FIGURE 24: Hexapod walking system.

compared with the analog approaches, but the disadvantages of hardware complexity and bus width [53].

\section{CMOS DT-CNN Implementation}

The whole system has been designed on BMR as follows [54]. The proposed design is a simplified version of the CPG (Figure 21). This design corresponds to the fact that in most CPGs, the majority of interconnections between neurons are inhibitory [46]. The output saturation of the activation function corresponds to the maximum IPMC actuation (bending angle).

The CPG CNN structure shown in Figure 21 is built for the insect tripod gait. Each population of neurons is connected to a group of legs (A or $\mathrm{B}$ ). Thus, the first one controls the first tripod $\left(\mathrm{L}_{1} \mathrm{R}_{2} \mathrm{~L}_{3}\right)$ and the second controls the other tripod $\left(\mathrm{R}_{1} \mathrm{~L}_{2} \mathrm{R}_{3}\right)$. This pair of mutually inhibited cells, with a suitable choice of the synaptic weights, is characterized by an antiphase synchronization of the activities of the two cells. The cells are implemented on FPGA using DA (Figure 22) with the architecture proposed in [54].

Each memory contains only the partial products for three coefficients. This configuration reduces storage by $95 \%$ in the direct implementation. The accumulator in DA performs add and shift operations.

\section{Control System}

The BMR body is the support for the current microlegs. The leg configuration on the body is an important consideration for driving the robot in any direction (Figure 23). In particular, the electrical configuration of the connections shown in Figure 23(b) of the legs is designed so as to produce a walking pattern. Experimental results show that the halfperiod of stance does not need any power consumption, while the other legs are in swing period (maximal current consumption). IPMC elasticity propels the BMR body. The

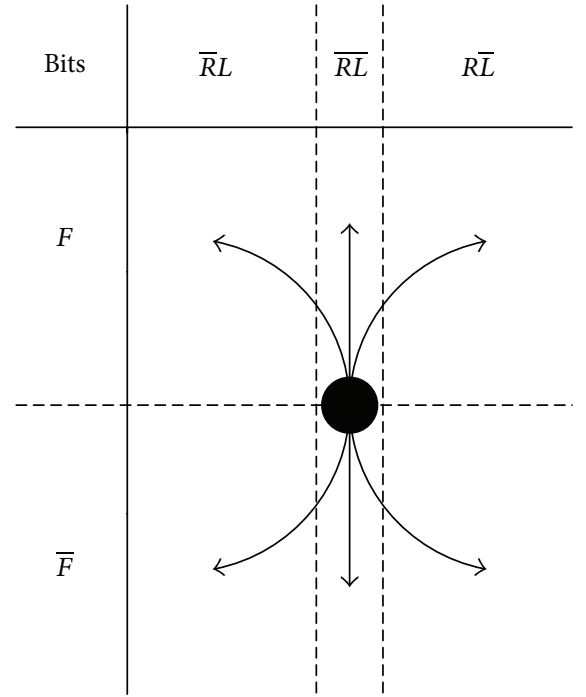

FIGURE 25: BMR possible direction control by PWM modulation.

bending angle decreases or increases the stance, and the swing time produces the BMR turn.

DT-CNN architecture for CPG has been included in the previous BMR control system to improve adaptability to this environment. The DT-CNN module controls the moore finite state machine (FSM) with GA and GB bits, the two tripod groups. DT-CNN has two inputs and five outputs. FSM is designed with eight states, and the output is controlled by the PWM as depicted in Figure 24. The MSB bits, R (right) and $\mathrm{L}$ (left), control the PWM width, while the ten bits, including LSB $\alpha$ bits, are proportional to the bending angle of the fiber IPMC actuator.

The BMR will move forward when $\mathrm{R}$ and $\mathrm{L}$ are set to zero. PWM width will be the same for the left and right legs as shown in Figure 25. In addition, it can turn the same way as an insect when the width of the PWM is changed for the left 


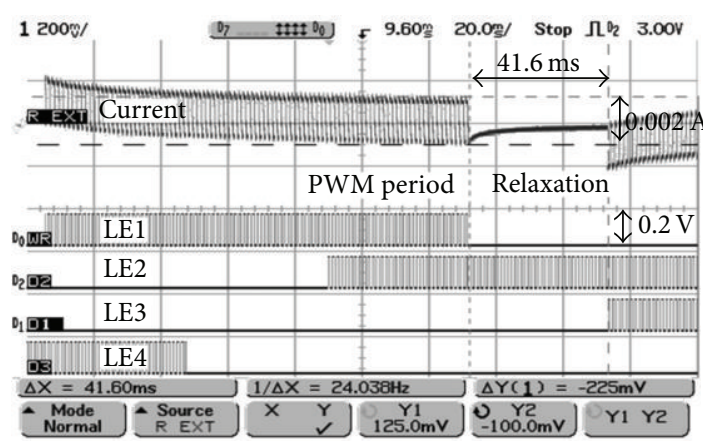

FIGURE 26: Applied PWM for LE1 to LE4 and current consumption for LE1 and LE3.

or right legs. Only three bits, stored in the microcontroller LEG register, are used to control the direction of the robot. Only these bits can be modified by user algorithms.

The overall system is coded as a driver in VHDL and is tested on FPGA. Currently, the PWM period is fixed in the PWM module. Experimental results show that a frequency of $763 \mathrm{~Hz}$ and $24 \mathrm{~Hz}$ for PWM and FSM, respectively, can drive one BMR leg. A half-period of stance is calculated at about 0.042 seconds (maximum bending to relaxation position). Figure 26 shows the FSM signal from the FPGA and current consumption with timing parameters. In future work, the module will be included in the microcontroller.

\section{Conclusion}

Microleg fabrication is an important challenge. It requires microscopic handling and specific instrumentation. Also, a failure in the procedure and a bad handling could damage the IPMC surface. Indeed, the distribution of the metal particles added on the IEM is very sensitive to the conditions of treatment, impregnation, and reduction. Microleg dimensions, according to the new manufacturing process worked out in this paper, make it possible to carry out a millimeter-size microrobot. Furthermore, the configuration of the electrodes confers 2,5 degrees of freedom. Thus, a hexapod robot will have 15 degrees of freedom unlike insects, which have at least 18 degrees of freedom. Using a micropump with a trust vector control improves maneuverability of the BMR.

Thereafter, several essential IPMC physical properties were given to drive 15 DOF hexapod BioMicroRobots. To measure these characteristics adequately, a very particular assembly was carried out. Inter alia, it was possible to show, without any doubt and using a laser vibrometer, that there is a linear relationship between the IPMC displacement rate and PWM report/ratio of cycle (duty cycle). It was also possible to measure the resonance frequency coupled with a heavy fluid. The minimum frequency for eliminating water electrolysis when the voltage is higher than the decomposition potential was also studied. The four methods for reducing water electrolysis explained in this paper are as follows:

(1) Using alkyl ammonium like TBA allow decreasing electrolysis and the relaxation effect with the Nafion membrane;
(2) keeping frequency higher than $30 \mathrm{~Hz}$, the limit of molecule diffusion through the electrode membrane;

(3) obtaining a high overpotential with better electrodes or keeping applied voltage lower than the decomposition potential;

(4) using bipolar current source to control the charge density and charge balance injected inside IPMC. It may be done by active and passive waveforms for the two phases of asymmetrical biphasic stimuli.

The evidence of linearity between the PWM displacement rate and the PWM duty cycle is a relationship, which will be very important for controlling the BMR in all directions like an insect. Moreover, the electromechanical characteristics provided in this paper will allow developing a control method for each microleg. In effect, power consumption and leg degree of freedom numbers are two of the many restrictive characteristics to be observed at the time of system design for autonomous robots.

Obviously, other interesting applications can benefit from the process, which will be developed. It is possible to envisage active electrodes for the mechanical stimulation in human implant prostheses. Indeed, these electrodes could stimulate the sensitive receivers not only electrically, but also mechanically.

Lastly, a new artificial locomotion servo-system with discrete time cellular neural networks (DT-CNN) has been developed for a bioinspired hexapod BMR. Using a state machine and PWM modulation to drive the actuators, the robot can move in any direction similarly to an insect. An overview of the robot central pattern generator (CPG) in accordance with the insect displacement principle has been demonstrated. MTA is used to perform a particular locomotion type with autowave generation. The control system can be structured as a digital control system done by CNNs generating the tripod locomotion pattern. Leg displacement is controlled by CPG as a function of the reflex sensor and the user algorithm in the microcontroller.

\section{Acknowledgments}

This work was partly supported by Groupe d'acoustique et vibrations de l'Université de Sherbrooke (GAUS) and Institut des Matériaux et des Systèmes Intelligents (IMSI). The author would like to thank Pierre Magny and Irène Levesque for their advice and suggestions for taking SEM images of the composite. As well, The author would like to thank Magella Tremblay and Patrice Masson for the use of their laboratory instrumentations.

\section{References}

[1] A. Bourjault and N. Chaillet, La Microrobotique, Hermes Sciences Publication, Paris, France, 2002.

[2] K. E. Drexler, Engines of Creation: The Coming Aera of Nanotechnology, Anchor Press/Doubleday, Garden City, NY, USA, 1990.

[3] A. Bonvilain and N. Chaillet, "Fabrication and experiment of microlegs for an insect-like microrobot," in Microrobotics and 
Microassembly III, vol. 4568 of Proceedings of SPIE, pp. 163-174, Boston, Mass, USA, October 2001.

[4] J. Enderle, S. Blanchard, and J. Bronzino, Introduction to Biomedical Engineering, Academic Press, San Diago, Calif, USA, 1999.

[5] S. Guo, L. Shi, N. Xiao, and K. Asaka, "A biomimetic underwater microrobot with multifunctional locomotion," Robotics and Autonomous Systems, vol. 60, no. 12, pp. 1472-1483, 2012.

[6] K. K. Ahna, D. Q. Truong, D. N. C. Nam, J. I. Yoon, and S. Yokotac, "Position control of ionic polymer metal composite actuator using quantitative feedback theory," Sensors and Actuators A, vol. 159, no. 2, pp. 204-212, 2010.

[7] L. Shi, S. Guo, and K. Asaka, "Modeling and experiments of IPMC actuators for the position precision of underwater legged microrobots," in Proceeding of the IEEE International Conference on Automation and Logistics, pp. 420-425, Zhengzhou, China, August 2012.

[8] M. Vahabi, E. Mehdizadeh, M. Kabganian, and F. Barazandeh, "Modelling of a novel in-pipe microrobot design with IPMC legs," Proceedings of the Institution of Mechanical Engineers I, vol. 225, no. 1, pp. 63-73, 2011.

[9] S. Martel, A. Saraswat, and I. Hunter, "Fundamentals of piezoceramic actuation for micrometer and sub-micrometer motions for the NanoWalker robot," in Microrobotics and Microassembly II, vol. 4194 of Proceedings of SPIE, pp. 82-93, Boston, Mass, USA, November 2000.

[10] M. Otis, M. Jarry, R. Bernier, R. Fontaine, H. Ménard, and P. Masson, "3D Modelization of an hexapod BioMicroRobot propulsion system with IPMC actuators," in Proceeding of the ReSMiQ's 1st Northeast Workshop on Circuits and Systems (NEWCAS '2003), Montreal, Canada, June 2003.

[11] M. Otis, R. Bernier, Y. Pasco et al., "Development of an hexapod BioMicRorobot with nafion-Pt IPMC microlegs," in Proceedings of the 25th Annual International Conference of the IEEE Engineering in Medicine and Biology Society, pp. 34233426, Cancun, Mexico, September 2003.

[12] K. Onishi, S. Sewa, K. Asaka, N. Fujiwara, and K. Oguro, "The effects of counter ions on characterization and performance of a solid polymer electrolyte actuator," Electrochimica Acta, vol. 46, no. 8, pp. 1233-1241, 2001.

[13] K. Onishi, S. Sewa, K. Asaka, N. Fujiwara, and K. Oguro, "Morphology of electrodes and bending response of the polymer electrolyte actuator," Electrochimica Acta, vol. 46, no. 5, pp. 737$743,2000$.

[14] M. Shahinpoor and K. J. Kim, "Effect of surface-electrode resistance on the performance of ionic polymer-metal composite (IPMC) artificial muscles," Smart Materials and Structures, vol. 9, no. 4, pp. 543-551, 2000.

[15] Y. Bar-Cohen, Electroactive Polymer (EAP) Actuators as Artificial Muscles, Reality, Potential, and Challenges, SPIE Press, Washington, DC, USA, 2001.

[16] G. Xie and T. Okada, "Pumping effects in water movement accompanying cation transport across nafion 117 membranes," Electrochimica Acta, vol. 41, no. 9, pp. 1569-1571, 1996.

[17] R. Kanno, S. Tadokoro, T. Takamori, and K. Oguro, "3dimensional dynamic model of ionic conducting polymer gel film (ICPF) actuator," in Proceedings of the IEEE International Conference on Systems, Man and Cybernetics, vol. 3, pp. 21792184, Beijing, China, October 1996.

[18] S. Guo, T. Nakamura, and T. Fukuda, "Micro active guide wire catheter system," in Proceedings of the IEEE International
Conference on Industrial Technology (ICIT '96), pp. 517-521, Shanghai, China, December 1996.

[19] S. B. Brummer and M. J. Turner, "Electrical stimulation with Pt electrodes. II. Estimation of maximum surface redox (theoretical non-gassing) limits," IEEE Transactions on Biomedical Engineering, vol. 24, no. 5, pp. 440-443, 1977.

[20] M. Shahinpoor and M. Mojarrad, "Soft actuators and artificial muscles," US patent 6109852, 2000.

[21] D. T. Sawyer, A. Sobkowiak, and J. L. Roberts, Electrochemistry for Chimists, John Wiley \& Sons, New York, NY, USA, 2nd edition, 1995.

[22] X. Bao, Y. Bar-Cohen, and S. Lih, "Measurements and macro models of ionomeric polymer-metal composites (IPMC)," in Smart Structures and Materials 2002: Electroactive Polymer Actuators and Devices (EAPAD), vol. 4695 of Proceedings of SPIE, pp. 220-227, San Diego, Calif, USA, March 2002.

[23] S. R. Taylor and E. Gileadi, "Physical interpretation of the Warburg impedance," Corrosion, vol. 51, no. 9, pp. 664-671, 1995.

[24] M. Shahinpoor and K. J. Kim, "Ionic polymer-metal composites: I. Fundamentals," Smart Materials and Structures, vol. 10, no. 4, pp. 819-833, 2001.

[25] Y. Bar-Cohen, S. Sherrit, and S. S. Lih, "Characterization of the electromechanical properties of EAP materials," in Smart Structures and Materials 2001: Electroactive Polymer Actuators and Devices, vol. 4329 of Proceedings of SPIE, pp. 319-327, Newport Beach, Calif, USA, March 2001.

[26] M. Jarry, M. Otis, H. Semmaoui, G. Haché, and R. Fontaine, "Core Control Circuit for Submillimiterrobot," in Proceedings of the 25th Annual International Conference of the IEEE Engineering in Medicine and Biology Society, pp. 3427-3430, Cancun, Mexico, September 2003.

[27] K. Mallavarapu and D. J. Leo, "Feedback control of the bending response of ionic polymer actuators," Journal of Intelligent Material Systems and Structures, vol. 12, no. 3, pp. 143-155, 2001.

[28] D. J. Gorman, Free Vibration Analysis of Beams and Shafts, John Wiley \& Sons, Toronto, Canada, 1995.

[29] R. J. Gilbert, Vibration des structures-Interaction avec les fluides-Sources d'excitations aléatoires, Éditions Eyrolles, Paris, France, 1998.

[30] S. Nemat-Nasser and Y. Wu, "Comparative experimental study of ionic polymer-metal composites with different backbone ionomers and in various cation forms," Journal of Applied Physics, vol. 93, no. 9, pp. 5255-5267, 2003.

[31] S. Sewa, K. Onishi, K. Asaka, N. Fujiwara, and K. Oguro, "Polymer actuator driven by ion current at low voltage, applied to catheter system," in Proceedings of the IEEE 11th Annual International Workshop on Micro Electro Mechanical Systems (MEMS '98), pp. 148-153, Heidelberg, Germany, January 1998.

[32] N. Fujiwara, K. Asaka, Y. Nishimura, K. Oguro, and E. Torikai, "Preparation of gold-solid polymer electrolyte composites as electric stimuli-responsive materials," Chemistry of Materials, vol. 12, no. 6, pp. 1750-1754, 2000.

[33] K. Asaka, N. Fujiwara, K. Oguro, K. Onishi, and S. Sewa, "State of water and ionic conductivity of solid polymer electrolyte membranes in relation to polymer actuators," Journal of Electroanalytical Chemistry, vol. 505, no. 1-2, pp. 24-32, 2001.

[34] A. L. Rollet, G. Gebel, J. P. Simonin, and P. Turq, "A SANS determination of the influence of external conditions on the nanostructure of nafion membrane," Journal of Polymer Science $B$, vol. 39, no. 5, pp. 548-558, 2001. 
[35] K. Oguro, N. Fujiwara, K. Asaka, K. Onishi, and S. Sewa, "Polymer electrolyte actuator with gold electrodes," in Smart Structures and Materials 1999: Electroactive Polymer Actuators and Devices, vol. 3669 of Proceedings of SPIE, pp. 64-71, Newport Beach, Calif, USA, March 1999.

[36] J. G. Webster, Medical Instrumentation Application and Design, John Wiley \& Sons, Toronto, Canada, 3rd edition, 1998.

[37] S. B. Brummer and L. S. Robblee, "Criteria for selecting electrodes for electrical stimulation: theoretical and practical considerations," Annals of the New York Academy of Sciences, vol. 405, pp. 159-171, 1983.

[38] J. M. Winter, Biomechanics and Neural Control of Posture and Movement, Springer, New York, NY, USA, 2000.

[39] H. Cruse, T. Kindermann, M. Schumm, J. Dean, and J. Schmitz, "Walknet-a biologically inspired network to control six-legged walking," Neural Networks, vol. 11, no. 7-8, pp. 1435-1447, 1998.

[40] P. Arena, H. Cruse, L. Fortuna, M. Frasca, and L. Patané, "A cellular nonlinear approach to decentralized locomotion control of the stick insect," in Proceedings of the IEEE International Symposium on Circuits and Systems, pp. IV-165-IV-168, May 2002.

[41] H. Cruse, "What mechanisms coordinate leg movement in walking arthropods?” Trends in Neurosciences, vol. 13, no. 1, pp. 15-21, 1990.

[42] J. Gray, Animal Locomotion, Great Britain, London and Beccles, edited by R. Carrington, The World Naturalist, 1968.

[43] L. H. Ting, R. Blickhan, and R. J. Full, "Dynamic and static stability in hexapedal runners," Journal of Experimental Biology, vol. 197, pp. 251-269, 1994.

[44] D. L. Jindrich and R. J. Full, "Many-legged maneuverability: dynamics of turning in hexapods," Journal of Experimental Biology, vol. 202, no. 12, pp. 1603-1623, 1999.

[45] P. S. Stein, "Motor systems, with specific reference to the control of locomotion," Annual Review of Neuroscience, vol. 1, pp. 61-81, 1978.

[46] K. G. Pearson, "Common principles of motor control in vertebrates and invertebrates," Annual Review of Neuroscience, vol. 16, pp. 265-297, 1993.

[47] P. Arena and L. Fortuna, "Reaction-diffusion CNN algorithms to generate and control artificial locomotion," IEEE Transactions on Circuits and Systems I, vol. 46, no. 2, pp. 253-260, 1999.

[48] P. Arena, L. Fortuna, and M. Branciforte, "Realization of a reaction-diffusion $\mathrm{CNN}$ algorithm for locomotion control in a hexapode robot," Journal of VLSI Signal Processing Systems for Signal, Image, and Video Technology, vol. 23, no. 2, pp. 267-280, 1999.

[49] P. Arena, L. Fortuna, M. Frasca, and C. Marchese, "MultiTemplate Approach to artificial locomotion control," in Proceedings of the IEEE International Symposium on Circuits and Systems (ISCAS '01), vol. 2, pp. 37-40, May 2001.

[50] L. O. Chua and L. Yang, "Cellular neural networks: theory," IEEE Transactions on Circuits and Systems, vol. 35, no. 10, pp. 12571272, 1988.

[51] A. Rodriguez-Vazquez, S. Espejo, R. Dominguez-Castro, J. L. Huertas, and E. Sanchez-Sinencio, "Current-mode techniques for the implementation of continuous- and discrete-time cellular neural networks," IEEE Transactions on Circuits and Systems II, vol. 40, no. 3, pp. 132-146, 1993.

[52] G. F. D. Betta, S. Graffi, Z. M. Kovacs, and G. Masetti, "CMOS implementation of an analogically programmable cellular neural network," IEEE Transactions on Circuits and Systems II, vol. 40, no. 3, pp. 206-215, 1993.
[53] S. Park, J. Lim, and S. Chae, "Digital implementation of discretetime cellular neural networks with distributed arithmetic," in Proceedings of the IEEE International Conference on Neural Networks, vol. 2, pp. 959-963, June 1997.

[54] P. Arena, L. Fortuna, and M. Frasca, "Attitude control in walking hexapod robots: an analogic spatio-temporal approach," International Journal of Circuit Theory and Applications, vol. 30, no. 2-3, pp. 349-362, 2002. 

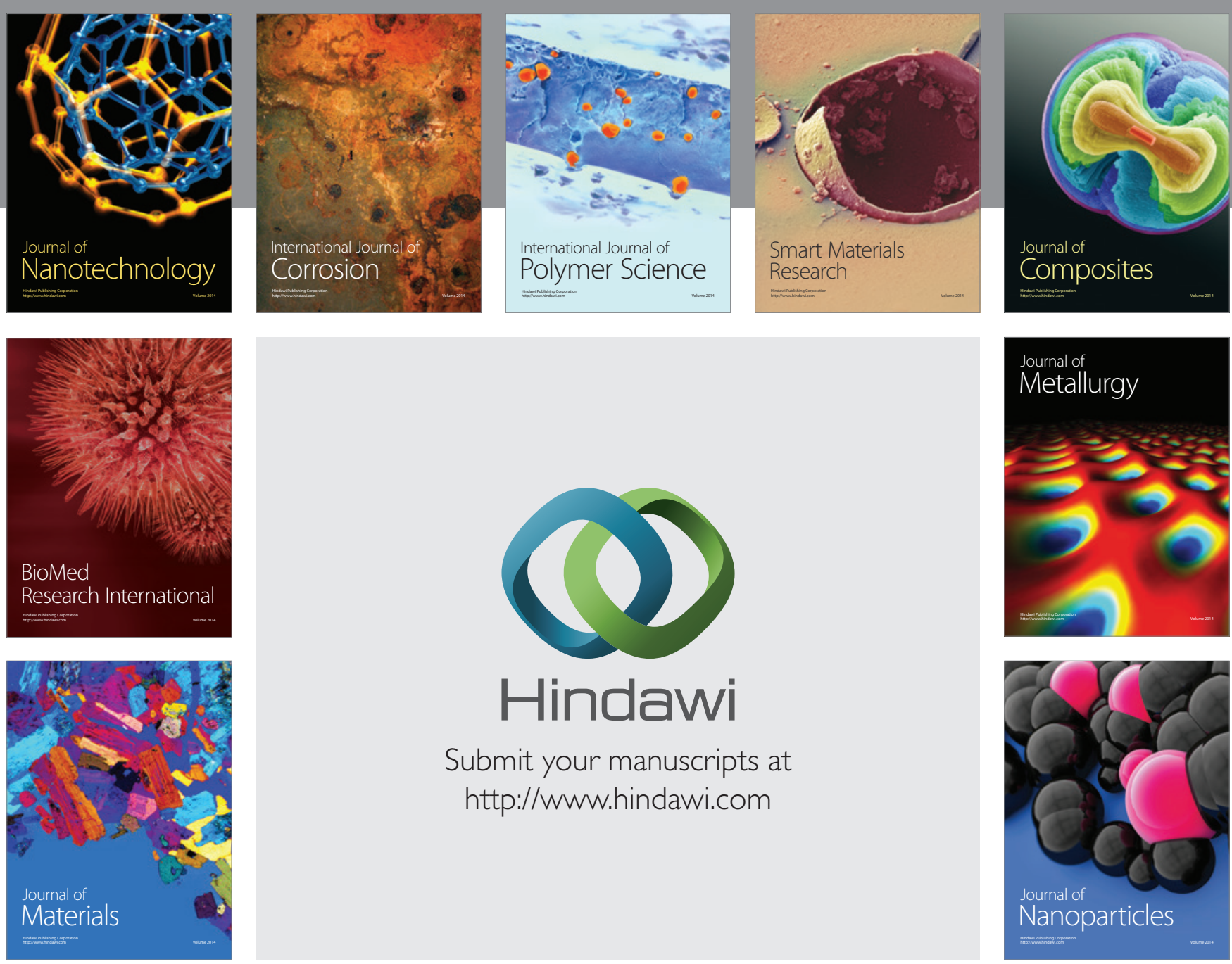

Submit your manuscripts at http://www.hindawi.com
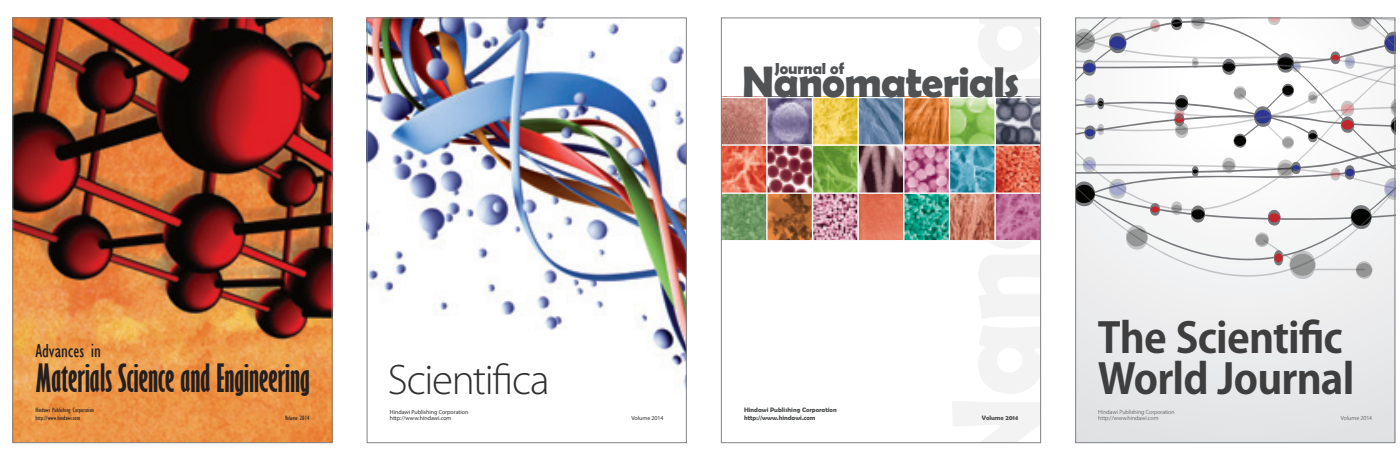

\section{The Scientific World Journal}
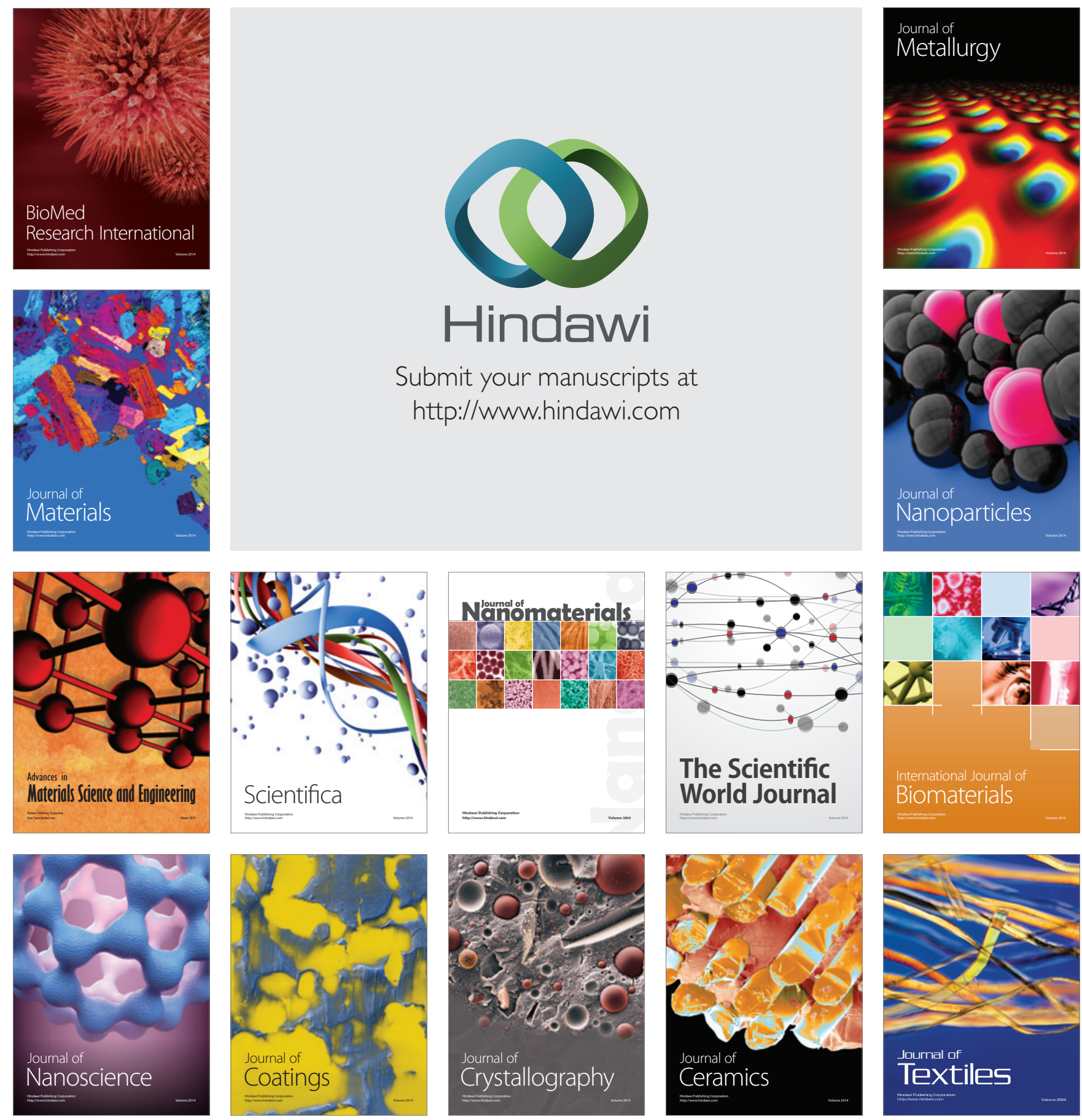